\title{
Nickel-Catalyzed Homocoupling of (Z)- $\beta$-lodoenol Esters: Stereoselective Access to (Z,Z)-Buta-1,3-diene-1,4-diyl Diesters
}

\author{
Javier Francos \\ Victorio Cadierno* \\ Laboratorio de Compuestos Organometálicos y Catálisis \\ (Unidad Asociada al CSIC), Centro de Innovación en Química \\ Avanzada (ORFEO-CINQA), Departamento de Química \\ Orgánica e Inorgánica, IUQOEM, Universidad de Oviedo, Julián \\ Clavería 8, E-33006 Oviedo, Spain \\ vcm@uniovi.es \\ Dedicated to Prof. Pablo Espinet on the occasion of his $70^{\text {th }}$ \\ birthday
}

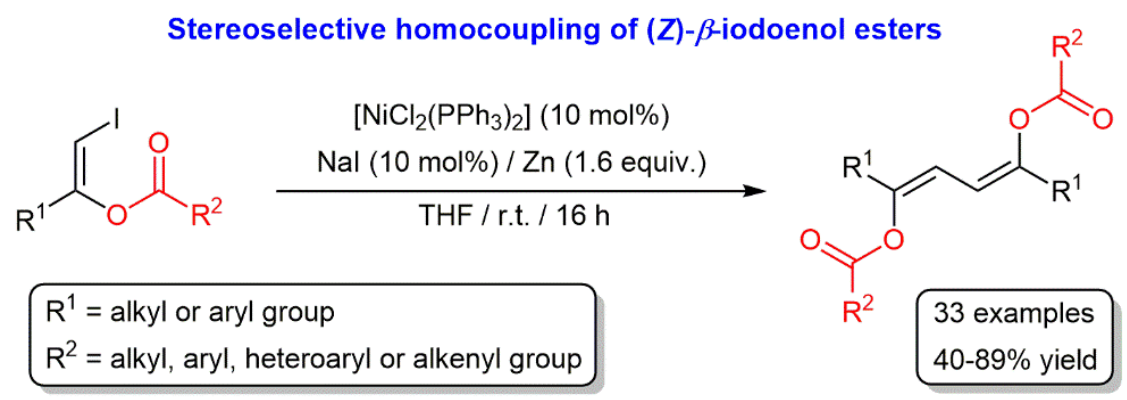

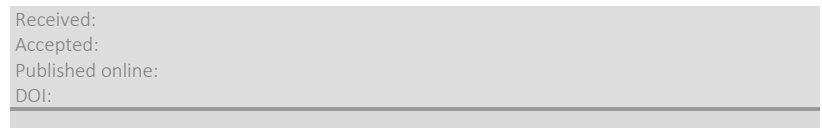

Abstract A straightforward and broad scope procedure to obtain symmetrically substituted buta-1,3-diene-1,4-diyl diesters, based on the homocoupling of the corresponding (Z)- $\beta$-iodoenol esters, is presented. It involves the use of a catalytic system composed of $\left[\mathrm{NiCl}_{2}\left(\mathrm{PPh}_{3}\right)_{2}\right](10 \mathrm{~mol} \%)$, $\mathrm{Nal}(10 \mathrm{~mol} \%)$ and excess of $\mathrm{Zn}$ dust. The reactions proceed in THF at r.t. with exquisite preservation of the stereochemistry of the $\mathrm{C}=\mathrm{C}$ bond of the starting iodo-olefins, thus leading to the final dienes as the corresponding $Z, Z$ stereoisomers exclusively.

Key words homocoupling reactions, nickel, iodoalkenes, buta-1,3-dienes, enol esters, diesters

Enol esters A are an important class of compounds widely employed as substrates and intermediates in organic chemistry. ${ }^{1}$ In particular, much attention has been devoted to the asymmetric hydrogenation of these molecules, since the resulting saturated esters $\mathbf{B}$ can be easily converted into synthetically relevant chiral alcohols $\mathbf{C}$ through a trivial deacylation process (Scheme 1). ${ }^{2}$

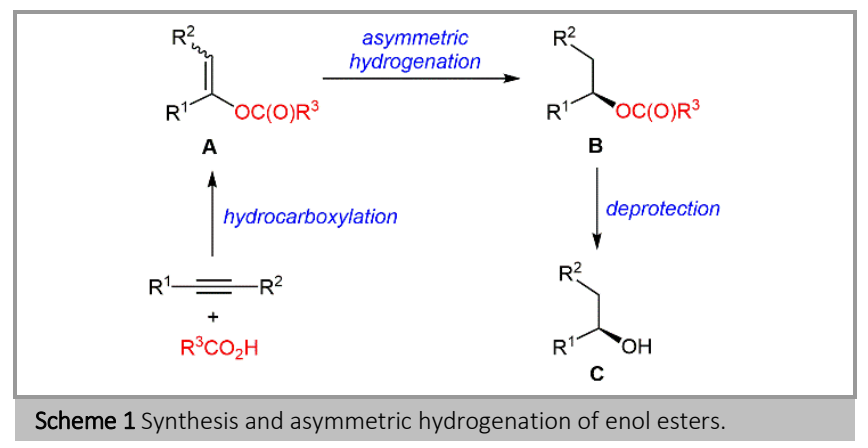

Among the different methods currently available for the synthesis of enol esters $\mathbf{A},{ }^{3}$ the addition of carboxylic acids to alkynes catalyzed by transition metal complexes is probably the most versatile and appealing one, due to its complete atom economy and substrates availability (Scheme 1).4,5 In this regard, in the context of our studies on this type of hydrocarboxylation processes, ${ }^{6}$ we recently developed a wide scope procedure for the synthesis of $(Z)$ - $\beta$-iodoenol esters $\mathbf{1}$ through the regio- and stereoselective intermolecular addition of carboxylic acids to iodoalkynes catalyzed by gold(I) (Scheme 2). ${ }^{6 \mathrm{~d}, \mathrm{f}, \mathrm{g}}$ Compounds $\mathbf{1}$ proved to be useful starting materials for the preparation of a large variety of stereochemically defined $\beta$ aryl-vinyl esters $2^{6 \mathrm{~d}, \mathrm{~g}}$ and enynyl esters $3^{6 \mathrm{f}}$ via Suzuki and Sonogashira cross-coupling reactions, respectively (Scheme 2). In addition, in collaboration with Pizzano's group (IIQ-Seville), we also studied the asymmetric hydrogenation of 2 using $\mathrm{Rh}$ catalysts, which allowed the access to the corresponding chiral homobenzylic esters with high enantioselectivity levels.6d,g

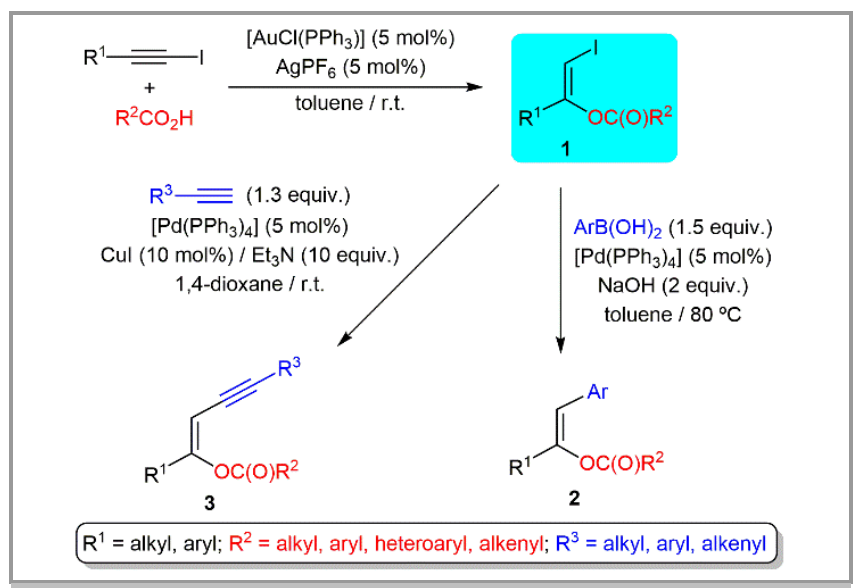

Scheme 2 Synthesis and palladium-catalyzed C-C couplings of (Z)- $\beta$-iodoenol esters 1. 
In this work, the synthetic utility of the (Z)- $\beta$-iodoenol esters $\mathbf{1}$ is further demonstrated with their successful involvement in homocoupling reactions to generate symmetrically substituted $(Z, Z)$-buta-1,3-diene-1,4-diyl diesters 4 (Figure 1). ${ }^{7}$ This type of conjugated dienes are scarcely represented in the literature. In fact, to the best of our knowledge, only five examples are currently known, featuring only one of them the same stereochemistry as compounds 4 (Figure 1 ). ${ }^{8}$

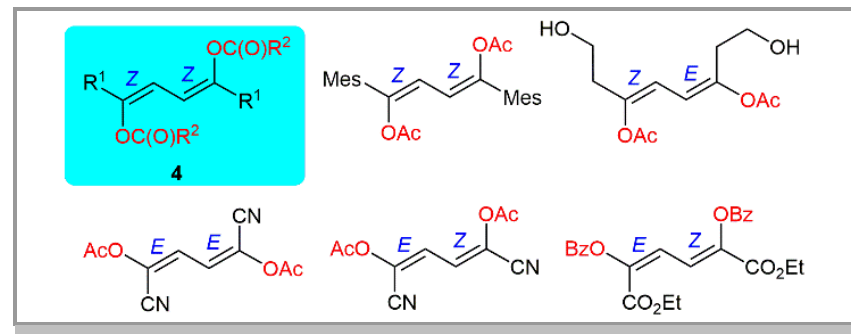

Figure 1 The generic structure of compounds 4 and that of related buta-1,3dienes reported in the literature.

On the other hand, we would like also to stress that, as we have very recently communicated employing a small family of alkyl-substituted diacetates (Scheme 3), the asymmetric hydrogenation of dienes $\mathbf{4}$ can open new routes for the synthesis of $C_{2}$ symmetric 1,4-diols, key building blocks in the preparation of a broad range of chiral heterocycles. ${ }^{9}$ Herein, a full report on the preparation of $(Z, Z)$-buta-1,3-diene-1,4-diyl diesters 4 is presented.

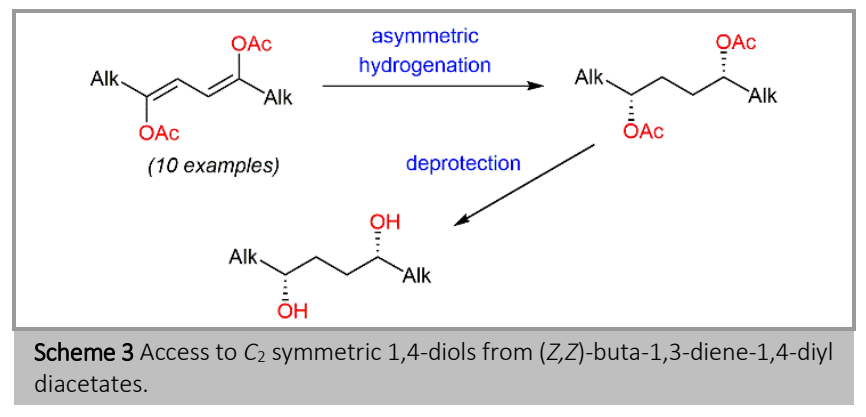

Initial attempts to promote the homocoupling of the $(Z)-\beta$ iodoenol esters 1 with copper(I) thiophene-2-carboxylate (CuTC), ${ }^{10}$ a reagent with proven effectiveness in reductive homocoupling reactions of aryl, heteroaryl and alkenyl halides, ${ }^{11}$ failed. Thus, regardless of the stoichiometry, solvent and temperature employed, the starting iodoolefins were recovered unchanged. Different palladium-based methodologies described in the literature were also tested with little success. ${ }^{12}$ Among them, only the one developed by Eddarir and Rolando led to the desired 1,3-dienes. ${ }^{12 \mathrm{~b}}$ However, as shown in Scheme 4 for the representative substrates 1a,b, the yields were very low and all attempts to improve them by changing the reaction parameters (solvent, temperature, time, and amounts of reagents) were unsuccessful.

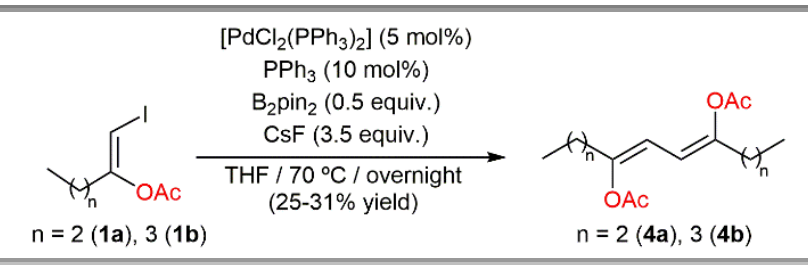

Scheme $4 \mathrm{Pd}$-catalyzed homocoupling of the (Z)- $\beta$-iodoenol esters $1 \mathrm{a}, \mathrm{b}$; $\mathrm{B}_{2}$ pin $_{2}=$ bis(pinacolato $)$ diboron.

The use of stoichiometric or catalytic amounts of zero-valent nickel complexes for homocoupling reactions of alkenyl halides is well documented, and this methodology is the one that led to the best results in the present case. ${ }^{13}$ Thus, as shown in Table 1 , the treatment of a THF solution of (Z)-1-iodopent-1-en-2-yl acetate (1a) with $30 \mathrm{~mol} \%$ of $\left[\mathrm{NiCl}_{2}\left(\mathrm{PPh}_{3}\right)_{2}\right]$, in the presence of $\mathrm{Zn}$ powder (2 equiv.) as the reductant and ${ }^{\mathrm{n}} \mathrm{Bu}_{4} \mathrm{NI}$ (1 equiv.) as an additive to assist the reduction of $\mathrm{Ni}(\mathrm{II})$ to $\mathrm{Ni}(0),{ }^{13, \mathrm{f}}$ for $16 \mathrm{~h}$ resulted in the clean formation of the desired diene $\mathbf{4 a}$, which was isolated in $67 \%$ yield after appropriate chromatographic work-up (entry 1). Interestingly, the reaction gives $4 \mathbf{4 a}$ as the corresponding $Z, Z$ isomer exclusively, indicating that $Z \rightarrow E$ isomerization of the starting iodoolefin does not take place along the process. ${ }^{13 \mathrm{f}}$ Reduction of the nickel loading to $10 \mathrm{~mol} \%$ led practically to the same result (entry 2), while the use of only $5 \mathrm{~mol} \%$ of $\left[\mathrm{NiCl}_{2}\left(\mathrm{PPh}_{3}\right)_{2}\right]$ resulted in a significant decrease in the effectiveness of the process (entry 3). Additional experiments also revealed the need to include the three components of the catalytic system for the reaction to proceed efficiently (entries 4-6). On the other hand, as shown in entry 7, the addition of extra $\mathrm{PPh}_{3}$ to the reaction medium did not lead to significant changes in the yield of $\mathbf{4 a} .{ }^{13 c, \mathrm{f}}$ Alternative nickel sources, i.e. $\mathrm{NiCl}_{2}$ and $\left[\mathrm{NiCl}_{2}\right.$ (glyme)], were also explored and resulted less effective than $\left[\mathrm{NiCl}_{2}\left(\mathrm{PPh}_{3}\right)_{2}\right]$ (entries 8-9). On the contrary, the use of inorganic iodide sources instead of $\mathrm{nBu}_{4} \mathrm{NI}$ allowed to improve the yield of 4a (entries 10-11). In particular, the best results were obtained with $\mathrm{NaI}$, which led to $\mathbf{4 a}$ in $>80 \%$ yield even when only $10 \mathrm{~mol} \%$ was added to the reaction medium (entries 11-14). As shown in entry 14, under these conditions, it was also possible to reduce the quantity of $\mathrm{Zn}$ employed $(82 \%$ yield). Finally, other solvents, such as toluene or $\mathrm{CH}_{2} \mathrm{Cl}_{2}$, were also screened but resulted less effective (entries 15-16 vs entry 14).

Table 1 Nickel-Catalyzed Homocoupling of the (Z)- $\beta$-lodoenol Acetate 1a: Optimization of the Reaction Conditions ${ }^{a}$

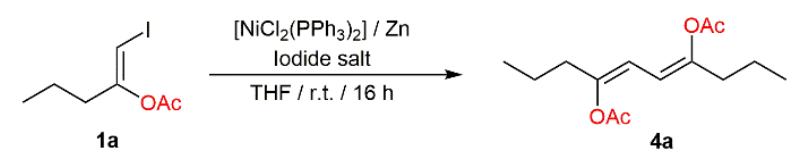

\begin{tabular}{|c|c|c|c|c|}
\hline Entry & {$\left[\mathrm{NiCl}_{2}\left(\mathrm{PPh}_{3}\right)_{2}\right]$} & lodide salt & $\mathrm{Zn}$ & Yield $^{b}$ \\
\hline 1 & 30 mol\% & ${ }^{n} \mathrm{Bu}_{4} \mathrm{NI}$ (1 equiv.) & 2 equiv. & $67 \%$ \\
\hline 2 & $10 \mathrm{~mol} \%$ & ${ }^{n} \mathrm{Bu}_{4} \mathrm{NI}$ (1 equiv.) & 2 equiv. & $65 \%$ \\
\hline 3 & 5 mol\% & ${ }^{n} \mathrm{Bu}_{4} \mathrm{NI}$ (1 equiv.) & 2 equiv. & $33 \%$ \\
\hline 4 & $10 \mathrm{~mol} \%$ & --- & 2 equiv. & $27 \%$ \\
\hline 5 & $10 \mathrm{~mol} \%$ & ${ }^{n} \mathrm{Bu}_{4} \mathrm{NI}$ (1 equiv.) & ---- & $0 \%$ \\
\hline 6 & ---- & ${ }^{\mathrm{n}} \mathrm{Bu}_{4} \mathrm{NI}$ (1 equiv.) & 2 equiv. & $0 \%$ \\
\hline $7^{c}$ & $10 \mathrm{~mol} \%$ & ${ }^{n} \mathrm{Bu}_{4} \mathrm{NI}$ (1 equiv.) & 2 equiv. & $63 \%$ \\
\hline $8^{d}$ & $10 \mathrm{~mol} \%$ & ${ }^{n} \mathrm{Bu}_{4} \mathrm{NI}$ (1 equiv.) & 2 equiv. & $48 \%$ \\
\hline$g^{e}$ & $10 \mathrm{~mol} \%$ & ${ }^{\mathrm{n} B \mathrm{u}_{4}} \mathrm{NI}$ (1 equiv.) & 2 equiv. & $62 \%$ \\
\hline 10 & 10 mol\% & KI (1 equiv.) & 2 equiv. & $75 \%$ \\
\hline 11 & 10 mol\% & Nal (1 equiv.) & 2 equiv. & $84 \%$ \\
\hline
\end{tabular}




\begin{tabular}{|c|c|c|c|c|c|}
\hline 12 & 10 mol\% & $\mathrm{Nal}(50 \mathrm{~mol} \%)$ & 2 equiv. & $84 \%$ & a Reactions performed under $\mathrm{Ar}$ atmosphere for $16 \mathrm{~h}$ employing $1 \mathrm{mmol}$ of $1 \mathrm{a}$ and \\
\hline 13 & $10 \mathrm{~mol} \%$ & $\mathrm{Nal}(10 \mathrm{~mol} \%)$ & 2 equiv. & $83 \%$ & $5 \mathrm{~mL}$ of anhydrous THF. ${ }^{b}$ Isolated yield after chromatographic work-up is given. ${ }^{c}$ \\
\hline 14 & $10 \mathrm{~mol} \%$ & Nal (10 mol\%) & 1.6 equiv. & $82 \%$ & Reaction performed in the presence of free $\mathrm{PPh}_{3}(20 \mathrm{~mol} \%)$. ${ }^{d}$ Reaction performed \\
\hline $15^{f}$ & $10 \mathrm{~mol} \%$ & Nal (10 mol\%) & 1.6 equiv. & $65 \%$ & 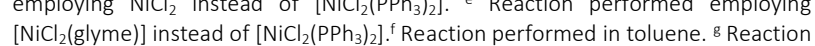 \\
\hline $16^{\mathrm{g}}$ & $10 \mathrm{~mol} \%$ & $\mathrm{Nal}(10 \mathrm{~mol} \%)$ & 1.6 equiv. & $47 \%$ & performed in $\mathrm{CH}_{2} \mathrm{Cl}_{2}$. \\
\hline
\end{tabular}

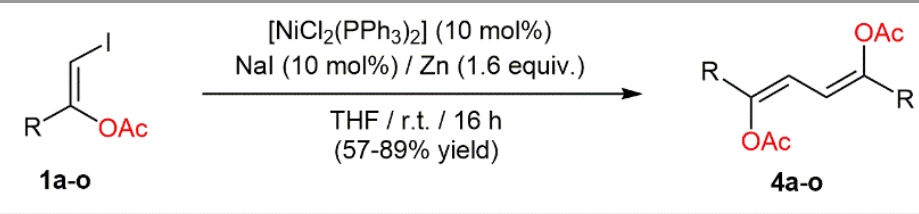<smiles>CC(C)CC/C(=C/C=C(/CCC(C)C)OC(C)(C)C)OC(C)(C)C</smiles>

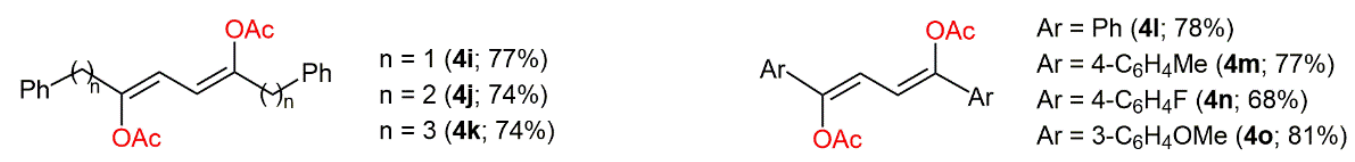

Scheme 5 Nickel-catalyzed homocoupling of (Z)- $\beta$-iodoenol acetates 1a-o. Isolated yields after column chromatography are given.

With the optimized reaction conditions in hand (entry 14 in Table 1), the scope of the process was next explored. In this regard, the behaviour of a series of $(Z)$ - $\beta$-iodoenol acetates $\mathbf{1} \mathbf{b}$ o was firstly investigated (Scheme 5). Thus, as observed for 1a, other iodoolefins containing alkyl and cycloalkyl substituents $\mathbf{1 b}-\mathbf{h}$ could be successfully converted into the corresponding buta-1,3-diene-1,4-diyl diacetates $\mathbf{4 b - h}$ in high yields (71-89\%). Good results were also obtained employing substrates bearing phenyl-substituted alkyl groups (1i-k) and those generated by addition of acetic acid to aromatic iodoalkynes (11-o), the resulting dienes 4i-o being isolated in $68-81 \%$ yield. Remarkably, all these homocoupling reactions proceeded with complete preservation of the stereochemistry of the $\mathrm{C}=\mathrm{C}$ bond of the starting iodoolefins $\mathbf{1}$, leading to compounds $\mathbf{4}$ as the corresponding $Z, Z$-stereoisomers exclusively. In this regard, the ${ }^{1} \mathrm{H}$ and ${ }^{13} \mathrm{C}$ NMR spectra confirmed the equivalence of the two $\mathrm{CH}=\mathrm{CR}(\mathrm{OAc})$ units of 4a-o, with singlet signals at $\delta_{\mathrm{H}}=5.58-6.64$ ppm and $\delta_{\mathrm{c}}=107.3-110.7(=\mathrm{CH})$ and 146.9-154.0 (=C) ppm being characteristic fingerprints of the symmetrically substituted 1,3-dienic units (see details in the Experimental Section). In addition, the molecular structure of $(Z, Z)-1,4-$ dicyclopentylbuta-1,3-diene-1,4-diyl diacetate (4g) was also determined by X-ray diffraction analysis, for which good quality crystals could be obtained by slow diffusion of hexane into a saturated solution of the compound in ethyl acetate. ${ }^{14}$ An ORTEP-type drawing of the molecule, along with selected bonding parameters, is shown in Figure 2. The C(3)-C(4) and $\mathrm{C}(4)$-C(4a) bond lengths observed $(1.330(2)$ and $1.449(3) \AA$, respectively) fit well with those of the parent unsubstituted 1,3butadiene molecule $(\mathrm{C}=\mathrm{C}=1.341 \AA \AA$ and $\mathrm{C}-\mathrm{C}=1.463 \AA$ A $) .{ }^{15}$

On the other hand, although all the reactions collected in Scheme 5 were systematically carried on a $1 \mathrm{mmol}$ scale, we would like to remark that they can be scaled up without major problems. As a representative example, a reaction conducted with (Z)-1-iodooct-1-en-2-yl acetate (1d) on a $10 \mathrm{mmol}$ scale
(2.96 g) allowed the preparation of $1.32 \mathrm{~g}$ (78\% yield) of $(Z, Z)$ hexadeca-7,9-diene-7,10-diyl diacetate (4d).

Finally, in order to assess in more detail the scope of the process, the homocoupling of a series of $(Z)$-1-iodohex-1-en-2yl esters 1p-ag, featuring different aliphatic, aromatic, heteroaromatic, heterocyclic and $\alpha, \beta$-unsaturated substituents on the carboxylate unit, was explored under identical experimental conditions (Scheme 6). To our delight, the catalytic reaction proved to be broadly applicable, and the novel 1,3-dienes 4p-ag could be synthesized with complete stereoselectivity in moderate to good yields (40-89\%).

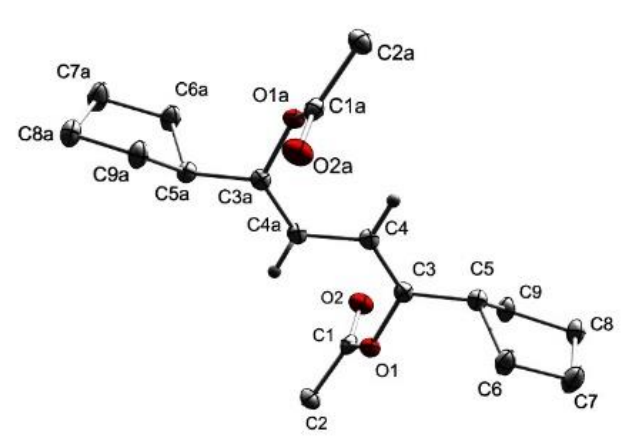

Figure 2 ORTEP-type view of the structure of diene $4 \mathrm{~g}$ showing the crystallographic labelling scheme. Hydrogen atoms, except those on C(4) and $C(4 a)$, have been omitted for clarity. Those atoms labelled with "a" are generated by symmetry. Thermal ellipsoids are drawn at $30 \%$ probability level. Selected bond lengths ( $\AA$ ): $C(1)-O(1) 1.365(2), C(1)-O(2) 1.199(2), C(1)$ $C(2) 1.488(2), C(3)-O(1) 1.410(2), C(3)-C(4) 1.330(2), C(3)-C(5) 1.493(2), C(4)-$ $C(4 a) 1.449(3)$. Selected bond angles ( $\left.{ }^{\circ}\right)$ : $O(1)-C(1)-O(2) 122.7(2), O(1)-C(1)-$ $C(2) 110.7(2), O(2)-C(1)-C(2) 126.6(2), C(1)-O(1)-C(3) 117.9(1), O(1)-C(3)-C(4)$ 119.3(1), $O(1)-C(3)-C(5)$ 114.3(1), $C(3)-C(5)-C(6)$ 114.5(1), $C(3)-C(5)-C(9)$ 116.1(1), $C(3)-C(4)-C(4 a) 125.3(2)$.

Concerning the mechanism of the present homocoupling process, we assume that it proceeds through the reaction pathway proposed by Takagi and co-workers (Scheme 7). ${ }^{16}$ It 
would involve the oxidative addition of the $\beta$-iodoenol ester to in situ formed $\mathrm{Ni}(0)$-phosphine species generated by reduction of $\left[\mathrm{NiCl}_{2}\left(\mathrm{PPh}_{3}\right)_{2}\right]$ with $\mathrm{Zn}$, a process that is facilitated by the $\mathrm{NaI}$ present in the reaction medium. Subsequent disproportionation of intermediate D thus formed, would generate the corresponding bis(alkenyl)-Ni(II) derivative $\mathbf{E}$ which undergo reductive elimination to form the final diene product. In this disproportionation step, $\left[\mathrm{NiI}_{2}\left(\mathrm{PPh}_{3}\right)_{2}\right]$ is also generated and reduced by the $\mathrm{Zn}$ powder to ensure the catalytic cycle.

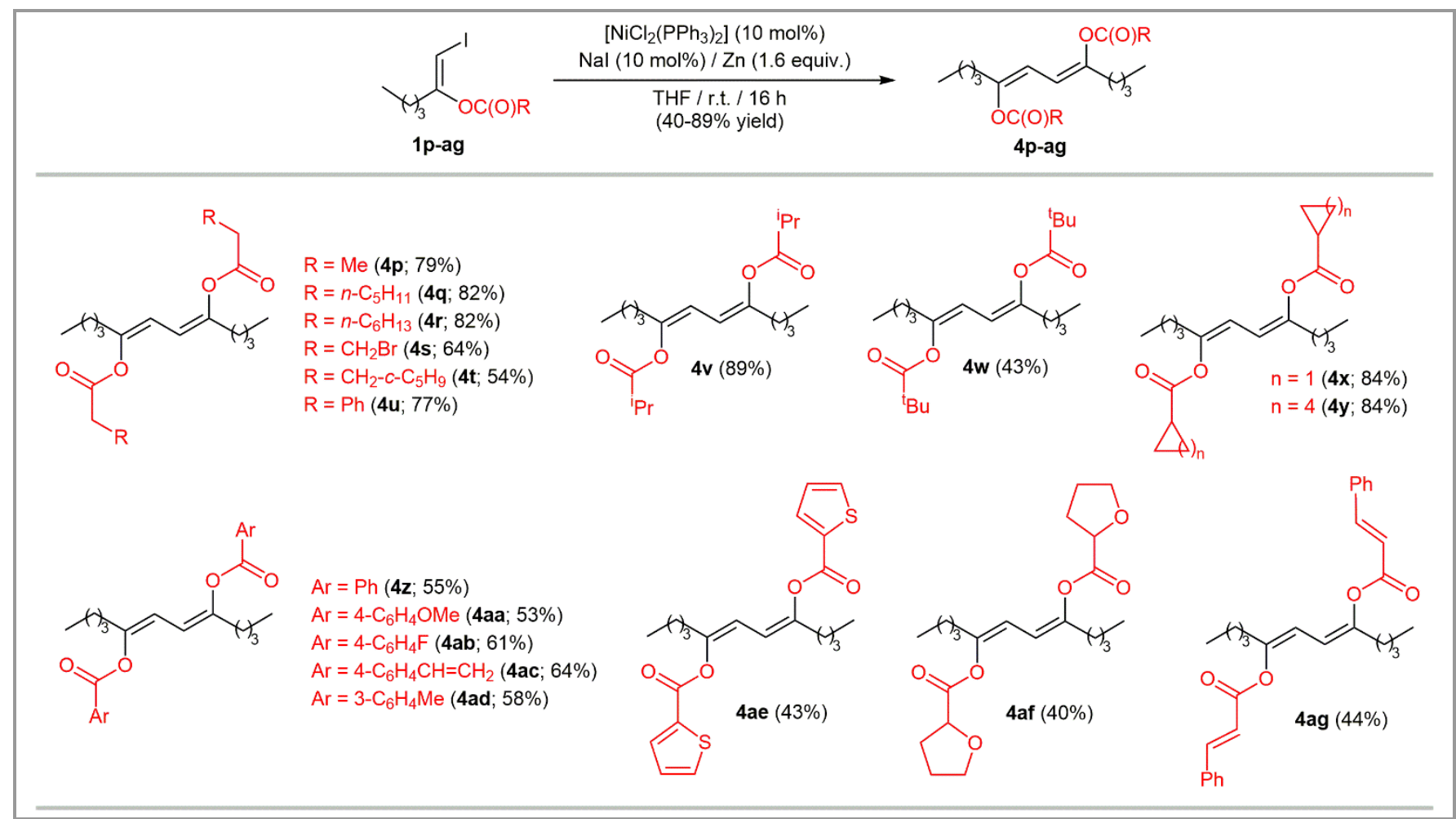

Scheme 6 Nickel-catalyzed homocoupling of (Z)- $\beta$-iodoenol esters 1: Scope of the process concerning the nature of the carboxylate group. Isolated yields after column chromatography are given.

Regarding the stereoselectivity of the process, as discussed by Takagi and co-workers, ${ }^{16}$ both the oxidative addition and reductive elimination steps are known to proceed with retention of the configuration of the alkenyl group. The exclusive formation of the Z,Z-stereoisomers implies that, unlike what is usually observed with other alkenyl halides, ${ }^{13,16}$ no isomerization of the $\mathrm{C}=\mathrm{C}$ bond is in the present case taking place at the disproportionation stage. Although we have not a conclusive explanation for this, we suspect that a potential interaction/coordination of the ester groups to $\mathrm{Ni}$ could be responsible of the high stereoselectivity observed.

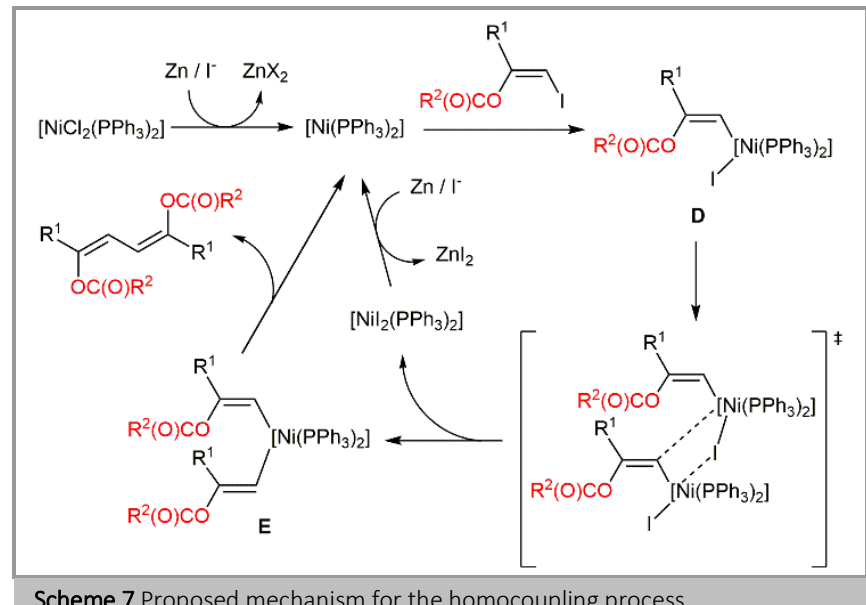

In summary, we have developed a general and inexpensive protocol for the preparation of buta-1,3-diene-1,4-diyl diesters, a family of underrepresented conjugated dienes, from readily accessible $(Z)$ - $\beta$-iodoenol esters. The catalytic homocoupling of the latter proceeded cleanly, and under mild conditions, in the presence of zero-valent nickel species generated in situ by combining $\left[\mathrm{NiCl}_{2}\left(\mathrm{PPh}_{3}\right)_{2}\right], \mathrm{NaI}$ and $\mathrm{Zn}$ dust. Exquisite preservation of the stereochemistry of the $\mathrm{C}=\mathrm{C}$ bond of the starting iodoolefins was in all the cases observed, the reactions leading to the 1,3-diene products in a complete stereospecific manner, and with isolated yields ranging from $40 \%$ to $89 \%$.

\section{The experimental section has no title; please leave this line here}

All reactions were performed under argon atmosphere using standard Schlenk-type techniques. Solvents were distilled under argon with sodium-benzophenone-ketyl (for $\mathrm{Et}_{2} \mathrm{O}$ and THF) or sodium (for hexanes). The (Z)- $\beta$-iodoenol ester 1 were synthesized by hydrocarboxylation of the corresponding iodoalkyne as previously described by us (Scheme 2). ${ }^{6 \mathrm{f}}$ Characterization data for compounds 10-y,aa-ag, not previously reported, have been included in the Supporting Information file. $\left[\mathrm{NiCl}_{2}\left(\mathrm{PPh}_{3}\right)_{2}\right]$ was prepared by following the method described in the literature. ${ }^{17}$ All other reagents were purchased from commercial suppliers and used as received. IR spectra were recorded on a PerkinElmer 1720-XFT spectrometer. NMR spectra were obtained on Bruker DPX-300 or AV-400 spectrometers. ${ }^{13} \mathrm{C}\left\{{ }^{1} \mathrm{H}\right\}$ and ${ }^{1} \mathrm{H}$ chemical shifts were referenced to the residual signal of deuterated solvent. All data are reported in ppm downfield from $\left(\mathrm{CH}_{3}\right)_{4} \mathrm{Si}$. For ${ }^{19} \mathrm{~F}\left\{{ }^{1} \mathrm{H}\right\}$ NMR spectra, the chemical shifts were referenced to the $\mathrm{CFCl}_{3}$ standard. All NMR measurements were carried out at $25^{\circ} \mathrm{C}$. HRMS data were obtained on a 
ESI-q-TOF Bruker Impact II mass spectrometer. For column chromatography, Merck silica gel 60 (230-400 mesh) was employed.

General Procedure for the Synthesis of $(Z, Z)$-Buta-1,3-diene-1,4-diyl diesters 4

A suspension of $\left[\mathrm{NiCl}_{2}\left(\mathrm{PPh}_{3}\right)_{2}\right](0.065 \mathrm{~g}, 0.1 \mathrm{mmol})$, NaI $(0.015 \mathrm{~g}, 0.1$ $\mathrm{mmol})$ and $\mathrm{Zn}$ dust $(0.105 \mathrm{~g}, 1.6 \mathrm{mmol})$ in THF $(5 \mathrm{~mL})$ was stirred at room temperature for $30 \mathrm{~min}$. After this time, a solution of the corresponding $(Z)$ - $\beta$-iodoenol ester 1 ( $1 \mathrm{mmol})$ in THF ( $2 \mathrm{~mL}$ ) was added and the resulting mixture further stirred at r.t. for $16 \mathrm{~h}$. The solvent was then removed under reduced pressure, and the crude reaction mixture directly purified by flash column chromatography over silica gel, using hexanes/diethyl ether (10:1) as eluent, to provide the desired products 4 .

\section{(Z,Z)-Deca-4,6-diene-4,7-diyl diacetate (4a)}

White solid; yield: $0.104 \mathrm{~g}$ (0.409 mmol; 82\%); mp 61-63 ${ }^{\circ} \mathrm{C}$.

IR (KBr): 2960, 2872, 1750, 1645, 1435, 1376, 1260, 1200, 1112, 1038, $1010,942,820,721,610,577,541,500,461 \mathrm{~cm}^{-1}$.

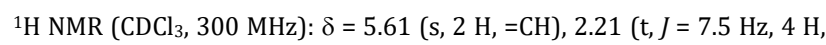
$\left.\mathrm{CH}_{2}\right), 2.15\left(\mathrm{~s}, 6 \mathrm{H}, \mathrm{CH}_{3}\right), 1.53-1.41\left(\mathrm{~m}, 4 \mathrm{H}, \mathrm{CH}_{2}\right), 0.88\left(\mathrm{t}, J=7.2 \mathrm{~Hz}, 6 \mathrm{H}, \mathrm{CH}_{3}\right)$. ${ }^{13} \mathrm{C} \mathrm{NMR}\left(\mathrm{CDCl}_{3}, 75 \mathrm{MHz}\right): \delta=168.5,149.6,109.2,35.6,20.7,19.9,13.5$. HRMS (ESI): $m / z[\mathrm{M}+\mathrm{Na}]^{+}$calcd for $\mathrm{C}_{14} \mathrm{H}_{22} \mathrm{O}_{4} \mathrm{Na}$ : 277.1410; found: 277.1406.

\section{(Z,Z)-Dodeca-5,7-diene-5,8-diyl diacetate (4b)}

White solid; yield: $0.126 \mathrm{~g}$ (0.446 mmol; 89\%); $\mathrm{mp} \mathrm{57-59}{ }^{\circ} \mathrm{C}$.

IR (KBr): 2954, 2870, 1760, 1647, 1430, 1371, 1200, 1099, 1024, 933, 878, $831,785,735,692,611,573,504,490 \mathrm{~cm}^{-1}$.

${ }^{1} \mathrm{H} \mathrm{NMR}\left(\mathrm{CDCl}_{3}, 400 \mathrm{MHz}\right): \delta=5.64(\mathrm{~s}, 2 \mathrm{H},=\mathrm{CH}), 2.26(\mathrm{t}, J=7.2 \mathrm{~Hz}, 4 \mathrm{H}$, $\left.\mathrm{CH}_{2}\right), 2.19\left(\mathrm{~s}, 6 \mathrm{H}, \mathrm{CH}_{3}\right), 1.44-1.40\left(\mathrm{~m}, 4 \mathrm{H}, \mathrm{CH}_{2}\right), 1.35-1.31\left(\mathrm{~m}, 4 \mathrm{H}, \mathrm{CH}_{2}\right)$, $0.90\left(\mathrm{t}, J=7.2 \mathrm{~Hz}, 6 \mathrm{H}, \mathrm{CH}_{3}\right)$.

${ }^{13} \mathrm{C} \mathrm{NMR}\left(\mathrm{CDCl}_{3}, 75 \mathrm{MHz}\right): \delta=168.6,149.9,109.0,33.3,28.7,22.1,20.8$, 13.8.

HRMS (ESI): $m / z$ [M+H] ${ }^{+}$calcd for $\mathrm{C}_{16} \mathrm{H}_{27} \mathrm{O}_{4}: 283.1904$; found: 283.1908 .

\section{(Z,Z)-Tetradeca-6,8-diene-6,9-diyl diacetate (4c)}

White solid; yield: $0.132 \mathrm{~g}$ (0.425 mmol; 85\%); mp 60-62 ${ }^{\circ} \mathrm{C}$.

IR (KBr): 2959, 2873, 1745, 1646, 1466, 1428, 1374, 1201, 1102, 1046, $1015,934,880,853,815,730,610,576,489 \mathrm{~cm}^{-1}$.

${ }^{1} \mathrm{H} \mathrm{NMR}\left(\mathrm{CDCl}_{3}, 300 \mathrm{MHz}\right): \delta=5.65(\mathrm{~s}, 2 \mathrm{H},=\mathrm{CH}), 2.27(\mathrm{t}, J=7.2 \mathrm{~Hz}, 4 \mathrm{H}$, $\left.\mathrm{CH}_{2}\right), 2.21\left(\mathrm{~s}, 6 \mathrm{H}, \mathrm{CH}_{3}\right), 1.48-1.43\left(\mathrm{~m}, 4 \mathrm{H}, \mathrm{CH}_{2}\right), 1.32-1.29\left(\mathrm{~m}, 8 \mathrm{H}, \mathrm{CH}_{2}\right)$, $0.90\left(\mathrm{t}, J=7.2 \mathrm{~Hz}, 6 \mathrm{H}, \mathrm{CH}_{3}\right)$.

${ }^{13} \mathrm{C} \mathrm{NMR}\left(\mathrm{CDCl}_{3}, 75 \mathrm{MHz}\right): \delta=168.6,149.9,109.1,33.6,31.2,26.3,22.4$, $20.8,14.0$.

HRMS (ESI): $m / z[\mathrm{M}+\mathrm{Na}]^{+}$calcd for $\mathrm{C}_{18} \mathrm{H}_{30} \mathrm{O}_{4} \mathrm{Na}$ : 333.2036; found: 333.2034.

\section{(Z,Z)-Hexadeca-7,9-diene-7,10-diyl diacetate (4d)}

White solid; yield: $0.135 \mathrm{~g}$ (0.399 mmol; 80\%); $\mathrm{mp} \mathrm{48-51}{ }^{\circ} \mathrm{C}$.

IR (KBr): 2925, 2857, 1754, 1643, 1438, 1374, 1201, 1130, 1018, 933, 871 , $809,722,622,578,554,465 \mathrm{~cm}^{-1}$.

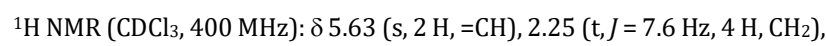
$2.19\left(\mathrm{~s}, 6 \mathrm{H}, \mathrm{CH}_{3}\right), 1.44-1.41\left(\mathrm{~m}, 4 \mathrm{H}, \mathrm{CH}_{2}\right), 1.27\left(\mathrm{br}, 12 \mathrm{H}, \mathrm{CH}_{2}\right), 0.88(\mathrm{t}, J=$ $\left.6.0 \mathrm{~Hz}, 6 \mathrm{H}, \mathrm{CH}_{3}\right)$.

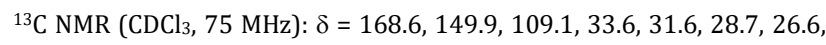
$22.5,20.8,14.0$.

HRMS (ESI): $m / z$ [M+Na] ${ }^{+}$calcd for $\mathrm{C}_{20} \mathrm{H}_{34} \mathrm{O}_{4} \mathrm{Na}$ : 361.2349; found: 361.2351.

(Z,Z)-2,11-Dimethyldodeca-5,7-diene-5,8-diyl diacetate (4e)
White solid; yield: $0.116 \mathrm{~g}(0.373 \mathrm{mmol}$; $75 \%)$; mp 65-67 ${ }^{\circ} \mathrm{C}$.

IR (KBr): 2952, 2864, 1747, 1650, 1470, 1441, 1372, 1261, 1216, 1131, $1098,1017,935,906,856,810,767,671,614,590,543,478 \mathrm{~cm}^{-1}$.

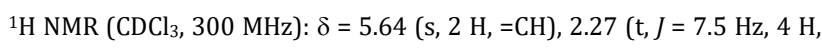
$\left.\mathrm{CH}_{2}\right), 2.21\left(\mathrm{~s}, 6 \mathrm{H}, \mathrm{CH}_{3}\right), 1.59-1.53(\mathrm{~m}, 2 \mathrm{H}, \mathrm{CH}), 1.38-1.30\left(\mathrm{~m}, 4 \mathrm{H}, \mathrm{CH}_{2}\right)$, $0.89\left(\mathrm{~d}, J=6.6 \mathrm{~Hz}, 12 \mathrm{H}, \mathrm{CH}_{3}\right)$.

${ }^{13} \mathrm{C} \mathrm{NMR}\left(\mathrm{CDCl}_{3}, 75 \mathrm{MHz}\right): \delta=168.6,150.1,108.9,35.6,31.6,27.6,22.4$, 20.8 .

HRMS (ESI): $m / z[\mathrm{M}+\mathrm{Na}]^{+}$calcd for $\mathrm{C}_{18} \mathrm{H}_{30} \mathrm{O}_{4} \mathrm{Na}$ : 333.2036; found: 333.2040 .

\section{(Z,Z)-1,4-Dicyclopropylbuta-1,3-diene-1,4-diyl diacetate (4f)}

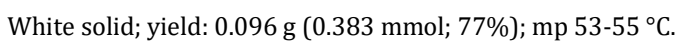

IR (KBr): 3095, 3019, 2929, 1751, 1635, 1559, 1436, 1374, 1261, 1194 , $1156,1043,1008,932,885,802,656,613,574,502 \mathrm{~cm}^{-1}$.

${ }^{1} \mathrm{H} \mathrm{NMR}\left(\mathrm{CDCl}_{3}, 300 \mathrm{MHz}\right): \delta 5.66(\mathrm{~s}, 2 \mathrm{H},=\mathrm{CH}), 2.21\left(\mathrm{~s}, 6 \mathrm{H}, \mathrm{CH}_{3}\right), 1.65-1.56$ (m, $2 \mathrm{H}, \mathrm{CH}), 0.72-0.66\left(\mathrm{~m}, 4 \mathrm{H}, \mathrm{CH}_{2}\right), 0.61-0.58\left(\mathrm{~m}, 4 \mathrm{H}, \mathrm{CH}_{2}\right)$.

${ }^{13} \mathrm{C} \mathrm{NMR}\left(\mathrm{CDCl}_{3}, 75 \mathrm{MHz}\right): \delta=168.6,149.8,108.3,20.6,14.3,5.7$.

HRMS (ESI): $m / z$ [M+Na] ${ }^{+}$calcd for $\mathrm{C}_{14} \mathrm{H}_{18} \mathrm{O}_{4} \mathrm{Na}$ : 273.1097; found: 273.1100 .

\section{(Z,Z)-1,4-Dicyclopentylbuta-1,3-diene-1,4-diyl diacetate (4g)}

White solid; yield: $0.124 \mathrm{~g}$ (0.405 mmol; 81\%); mp 60-62 ${ }^{\circ} \mathrm{C}$.

IR (KBr): 2961, 2870, 1751, 1638, 1436, 1376, 1207, 1137, 1017, 950, 890, $857,822,720,640,571,538,432 \mathrm{~cm}^{-1}$.

${ }^{1} \mathrm{H} \mathrm{NMR}\left(\mathrm{CDCl}_{3}, 300 \mathrm{MHz}\right): \delta 5.64(\mathrm{~s}, 2 \mathrm{H},=\mathrm{CH}), 2.75-2.64(\mathrm{~m}, 2 \mathrm{H}, \mathrm{CH}), 2.20$ (s, $\left.6 \mathrm{H} \mathrm{CH}_{3}\right), 1.81\left(\mathrm{br}, 4 \mathrm{H}, \mathrm{CH}_{2}\right), 1.66-1.46\left(\mathrm{~m}, 12 \mathrm{H}, \mathrm{CH}_{2}\right)$.

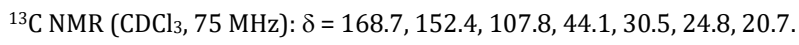

HRMS (ESI): $m / z[\mathrm{M}+\mathrm{Na}]^{+}$calcd for $\mathrm{C}_{18} \mathrm{H}_{26} \mathrm{O}_{4} \mathrm{Na}$ : 329.1723; found: 329.1726 .

\section{(Z,Z)-1,4-Dicyclohexylbuta-1,3-diene-1,4-diyl diacetate $(4 \mathrm{~h})$}

White solid; yield: $0.119 \mathrm{~g}(0.356 \mathrm{mmol} ; 71 \%)$; $\mathrm{mp} \mathrm{57-59}{ }^{\circ} \mathrm{C}$.

IR (KBr): 2950, 2873, 1753, 1635, 1440, 1377, 1215, 1200, 1140, 1009, $964,901,845,811,743,654,592,555,449 \mathrm{~cm}^{-1}$.

${ }^{1} \mathrm{H} \mathrm{NMR}\left(\mathrm{CDCl}_{3}, 300 \mathrm{MHz}\right): \delta 5.58(\mathrm{~s}, 2 \mathrm{H},=\mathrm{CH}), 2.22\left(\mathrm{~s}, 6 \mathrm{H}, \mathrm{CH}_{3}\right), 2.19-2.14$ (m, $2 \mathrm{H}, \mathrm{CH}), 1.89-1.66\left(\mathrm{~m}, 10 \mathrm{H}, \mathrm{CH}_{2}\right), 1.32-1.08\left(\mathrm{~m}, 10 \mathrm{H}, \mathrm{CH}_{2}\right)$.

${ }^{13} \mathrm{C} \mathrm{NMR}\left(\mathrm{CDCl}_{3}, 75 \mathrm{MHz}\right): \delta=168.7,154.0,107.3,42.0,30.6,26.0,25.9$, 20.7 .

HRMS (ESI): $m / z[\mathrm{M}+\mathrm{Na}]^{+}$calcd for $\mathrm{C}_{20} \mathrm{H}_{30} \mathrm{O}_{4} \mathrm{Na}$ : 357.2036; found: 357.2035 .

\section{(Z,Z)-1,6-Diphenylhexa-2,4-diene-2,5-diyl diacetate (4i)}

White solid; yield: $0.135 \mathrm{~g}$ (0.385 mmol; 77\%); $\mathrm{mp} 102-104{ }^{\circ} \mathrm{C}$.

IR (KBr): 3070, 3026, 2952, 1748, 1635, 1494, 1455, 1436, 1364, 1202, $1151,1114,1014,943,877,794,751,705,633,594,564,472 \mathrm{~cm}^{-1}$.

${ }^{1} \mathrm{H} \mathrm{NMR}\left(\mathrm{CDCl}_{3}, 300 \mathrm{MHz}\right): \delta$ 7.33-7.21 (m, $\left.10 \mathrm{H}, \mathrm{Ar}-\mathrm{H}\right), 5.68(\mathrm{~s}, 2 \mathrm{H},=\mathrm{CH})$, $3.63\left(\mathrm{~s}, 4 \mathrm{H}, \mathrm{CH}_{2}\right), 2.06\left(\mathrm{~s}, 6 \mathrm{H}, \mathrm{CH}_{3}\right)$.

${ }^{13} \mathrm{C} \mathrm{NMR}\left(\mathrm{CDCl}_{3}, 75 \mathrm{MHz}\right): \delta=168.5,149.2,136.6,129.2,128.5,126.8$, $110.7,39.9,20.6$.

HRMS (ESI): $m / z[\mathrm{M}+\mathrm{Na}]^{+}$calcd for $\mathrm{C}_{22} \mathrm{H}_{22} \mathrm{O}_{4} \mathrm{Na}$ : 373.1410; found: 373.1415 .

\section{(Z,Z)-1,8-Diphenylocta-3,5-diene-3,6-diyl diacetate (4j)}

White solid; yield: $0.140 \mathrm{~g}$ (0.370 mmol; 74\%); mp 97-99 ${ }^{\circ} \mathrm{C}$.

IR (KBr): 3061, 3023, 2898, 1737, 1651, 1602, 1495, 1431, 1372, 1358, $1266,1220,1102,1026,947,857,790,741,697,608,583,556,491 \mathrm{~cm}^{-1}$. 
${ }^{1} \mathrm{H} \mathrm{NMR}\left(\mathrm{CDCl}_{3}, 300 \mathrm{MHz}\right): \delta$ 7.38-7.19 (m, $\left.10 \mathrm{H}, \mathrm{Ar}-\mathrm{H}\right), 5.67(\mathrm{~s}, 2 \mathrm{H},=\mathrm{CH})$, $2.81\left(\mathrm{t}, J=7.8 \mathrm{~Hz}, 4 \mathrm{H}, \mathrm{CH}_{2}\right), 2.62\left(\mathrm{t}, J=7.8 \mathrm{~Hz}, 4 \mathrm{H}, \mathrm{CH}_{2}\right), 2.13\left(\mathrm{~s}, 6 \mathrm{H}, \mathrm{CH}_{3}\right)$. ${ }^{13} \mathrm{C} \mathrm{NMR}\left(\mathrm{CDCl}_{3}, 75 \mathrm{MHz}\right): \delta=168.6,149.3,140.9,128.4,128.3,126.1$, 109.6, 35.3, 33.1, 20.7.

HRMS (ESI): $m / z[M+N a]^{+}$calcd for $\mathrm{C}_{24} \mathrm{H}_{26} \mathrm{O}_{4} \mathrm{Na}$ : 401.1723; found: 401.1725 .

\section{(Z,Z)-1,10-Diphenyldeca-4,6-diene-4,7-diyl diacetate (4k)}

White solid; yield: $0.158 \mathrm{~g}(0.388 \mathrm{mmol}$; $78 \%)$; mp 107-109 ${ }^{\circ} \mathrm{C}$.

IR (KBr): 3023, 2944, 2860, 1746, 1644, 1602, 1498, 1462, 1369, 1204, $1121,1018,932,882,827,798,752,701,614,576,535,498,461 \mathrm{~cm}^{-1}$.

${ }^{1} \mathrm{H} \mathrm{NMR}\left(\mathrm{CDCl}_{3}, 300 \mathrm{MHz}\right): \delta$ 7.35-7.20 (m, $\left.10 \mathrm{H}, \mathrm{Ar}-\mathrm{H}\right), 5.70(\mathrm{t}, J=4.3 \mathrm{~Hz}$, $2 \mathrm{H},=\mathrm{CH}), 2.67\left(\mathrm{t}, J=7.4 \mathrm{~Hz}, 4 \mathrm{H}, \mathrm{CH}_{2}\right), 2.38-2.35\left(\mathrm{~m}, 4 \mathrm{H}, \mathrm{CH}_{2}\right), 2.22(\mathrm{~s}, 6$ $\left.\mathrm{H}, \mathrm{CH}_{3}\right), 1.85-1.82\left(\mathrm{~m}, 4 \mathrm{H}, \mathrm{CH}_{2}\right)$.

${ }^{13} \mathrm{C} \mathrm{NMR}\left(\mathrm{CDCl}_{3}, 75 \mathrm{MHz}\right): \delta=168.6,149.4,141.8,128.5,128.4,125.9$, 109.6, 35.1, 33.2, 28.2, 20.8

HRMS (ESI): $m / z[\mathrm{M}+\mathrm{Na}]^{+}$calcd for $\mathrm{C}_{26} \mathrm{H}_{30} \mathrm{O}_{4} \mathrm{Na}$ : 429.2036; found: 429.2040 .

\section{(Z,Z)-1,4-Diphenylbuta-1,3-diene-1,4-diyl diacetate (4l)}

Pale yellow solid; yield: 0.126 g (0.391 mmol; 78\%); mp 170-172 ${ }^{\circ} \mathrm{C}$.

IR (KBr): 3079, 2929, 1757, 1610, 1574, 1492, 1447, 1367, 1200, 1185, $1040,1025,860,755,687,626,569,518,442 \mathrm{~cm}^{-1}$.

${ }^{1} \mathrm{H} \mathrm{NMR}\left(\mathrm{CDCl}_{3}, 300 \mathrm{MHz}\right): \delta 7.50-7.36(\mathrm{~m}, 10 \mathrm{H}, \mathrm{Ar}-\mathrm{H}), 6.59(\mathrm{~s}, 2 \mathrm{H},=\mathrm{CH})$, $2.38\left(\mathrm{~s}, 6 \mathrm{H}, \mathrm{CH}_{3}\right)$.

${ }^{13} \mathrm{C} \mathrm{NMR}\left(\mathrm{CDCl}_{3}, 75 \mathrm{MHz}\right): \delta=168.6,148.1,134.6,128.9,128.7,124.7$, $110.4,20.7$.

HRMS (ESI): $m / z[\mathrm{M}+\mathrm{Na}]^{+}$calcd for $\mathrm{C}_{20} \mathrm{H}_{18} \mathrm{O}_{4} \mathrm{Na}$ : 345.1097; found: 345.1098.

\section{(Z,Z)-1,4-Bis(4-methylphenyl)buta-1,3-diene-1,4-diyl diacetate} (4m)

White solid; yield: $0.135 \mathrm{~g}$ (0.385 mmol; 77\%); mp $175-177^{\circ} \mathrm{C}$.

IR (KBr): 3070, 3032, 2921, 1769, 1612, 1504, 1371, 1210, 1194, 1175, $1034,1014,882,810,664,606,564,541,469 \mathrm{~cm}^{-1}$.

${ }^{1} \mathrm{H} \mathrm{NMR}\left(\mathrm{CDCl}_{3}, 300 \mathrm{MHz}\right): \delta 7.38(\mathrm{~d}, J=8.1 \mathrm{~Hz}, 4 \mathrm{H}, \mathrm{Ar}-\mathrm{H}), 7.19(\mathrm{~d}, J=8.1$ $\mathrm{Hz}, 4 \mathrm{H}, \mathrm{Ar}-\mathrm{H}), 6.53$ (s, $2 \mathrm{H},=\mathrm{CH}), 2.38\left(\mathrm{~s}, 12 \mathrm{H}, \mathrm{CH}_{3}\right)$.

${ }^{13} \mathrm{C} \mathrm{NMR}\left(\mathrm{CDCl}_{3}, 75 \mathrm{MHz}\right): \delta=168.6,147.9,138.9,131.8,129.4,124.6$ 109.6, 21.3, 20.8 .

HRMS (ESI): $m / z[\mathrm{M}+\mathrm{Na}]^{+}$calcd for $\mathrm{C}_{22} \mathrm{H}_{22} \mathrm{O}_{4} \mathrm{Na}$ : 373.1410; found: 373.1412 .

\section{(Z,Z)-1,4-Bis(4-fluorophenyl)buta-1,3-diene-1,4-diyl diacetate (4n)}

Pale yellow solid; yield: 0.122 g (0.340 mmol; 68\%); mp $164-166^{\circ} \mathrm{C}$.

IR (KBr): 3070, 2933, 1760, 1614, 1503, 1365, 1230, 1188, 1157, 1041, $913,878,836,820,753,609,566,533 \mathrm{~cm}^{-1}$.

${ }^{1} \mathrm{H}$ NMR (DMSO- $\left.d_{6}, 300 \mathrm{MHz}\right): \delta 7.67$ (br, $\left.4 \mathrm{H}, \mathrm{Ar}-\mathrm{H}\right), 7.24(\mathrm{br}, 4 \mathrm{H}, \mathrm{Ar}-\mathrm{H})$, $6.64(\mathrm{~s}, 2 \mathrm{H},=\mathrm{CH}), 2.43\left(\mathrm{~s}, 6 \mathrm{H}, \mathrm{CH}_{3}\right)$.

${ }^{13} \mathrm{C}$ NMR (DMSO- $\left.d_{6}, 101 \mathrm{MHz}\right): \delta=169.4,162.8(\mathrm{~d}, J=247.0 \mathrm{~Hz}), 146.9$ $130.9(\mathrm{~d}, J=2.8 \mathrm{~Hz}), 127.4(\mathrm{~d}, J=8.2 \mathrm{~Hz}), 116.1(\mathrm{~d}, J=21.8 \mathrm{~Hz}), 110.5,21.1$. ${ }^{19} \mathrm{~F}$ NMR (DMSO- $\left.d_{6}, 282 \mathrm{MHz}\right): \delta-112.4$.

HRMS (ESI): $m / z$ [M+Na] ${ }^{+}$calcd for $\mathrm{C}_{20} \mathrm{H}_{16} \mathrm{O}_{4} \mathrm{~F}_{2} \mathrm{Na}$ : 381.0909; found: 381.0913.

\section{(Z,Z)-1,4-Bis(3-methoxyphenyl)buta-1,3-diene-1,4-diyl diacetate} (40)

White solid; yield: $0.155 \mathrm{~g}$ (0.405 mmol; 81\%); mp 189-191 ${ }^{\circ} \mathrm{C}$.
IR (KBr): 3076, 2941, 2839, 1761, 1600, 1575, 1482, 1438, 1372, 1298, $1259,1210,1187,1033,917,842,781,682,580,506 \mathrm{~cm}^{-1}$.

${ }^{1} \mathrm{H}$ NMR ( $\left.\mathrm{CDCl}_{3}, 300 \mathrm{MHz}\right): \delta 7.31(\mathrm{t}, J=8.0 \mathrm{~Hz}, 2 \mathrm{H}, \mathrm{Ar}-\mathrm{H}), 7.10(\mathrm{~d}, J=8.0$ $\mathrm{Hz}, 2 \mathrm{H}, \mathrm{Ar}-\mathrm{H}$ ), 7.01 (d, $J=2.1 \mathrm{~Hz}, 2 \mathrm{H}, \mathrm{Ar}-\mathrm{H}$ ), 6.89 (dd, $J=8.0$ and $2.1 \mathrm{~Hz}, 2$ $\mathrm{H}, \mathrm{Ar}-\mathrm{H}), 6.57(\mathrm{~s}, 2 \mathrm{H},=\mathrm{CH}), 3.85\left(\mathrm{~s}, 6 \mathrm{H}, \mathrm{OCH}_{3}\right), 2.38\left(\mathrm{~s}, 6 \mathrm{H}, \mathrm{CH}_{3}\right)$.

${ }^{13} \mathrm{C} \mathrm{NMR}\left(\mathrm{CDCl}_{3}, 75 \mathrm{MHz}\right): \delta=168.6,159.8,147.9,136.1,129.8,117.3$, $113.9,110.9,110.6,55.3,20.8$.

HRMS (ESI): $m / z[\mathrm{M}+\mathrm{Na}]^{+}$calcd for $\mathrm{C}_{22} \mathrm{H}_{22} \mathrm{O}_{6} \mathrm{Na}$ : 405.1309; found: 405.1311 .

\section{(Z,Z)-Dodeca-5,7-diene-5,8-diyl dipropionate (4p)}

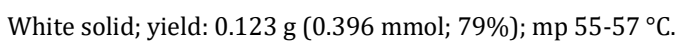

IR (KBr): 2984, 2939, 2872, 1749, 1645, 1454, 1413, 1364, 1303, 1151, $1130,931,869,822,807,731,558 \mathrm{~cm}^{-1}$.

${ }^{1} \mathrm{H} \mathrm{NMR}\left(\mathrm{CDCl}_{3}, 300 \mathrm{MHz}\right): \delta 5.62(\mathrm{~s}, 2 \mathrm{H},=\mathrm{CH}), 2.47\left(\mathrm{q}, J=7.5 \mathrm{~Hz}, 4 \mathrm{H}, \mathrm{CH}_{2}\right)$, $2.25\left(\mathrm{t}, J=7.2 \mathrm{~Hz}, 4 \mathrm{H}, \mathrm{CH}_{2}\right), 1.46-1.28\left(\mathrm{~m}, 8 \mathrm{H}_{\mathrm{CH}}\right), 1.21(\mathrm{t}, J=7.5 \mathrm{~Hz}, 6 \mathrm{H}$, $\left.\mathrm{CH}_{3}\right), 0.89\left(\mathrm{t}, J=7.2 \mathrm{~Hz}, 6 \mathrm{H}, \mathrm{CH}_{3}\right)$.

${ }^{13} \mathrm{C} \mathrm{NMR}\left(\mathrm{CDCl}_{3}, 75 \mathrm{MHz}\right): \delta=172.0,149.7,108.9,33.3,28.7,27.5,22.1$, 13.8, 9.2.

HRMS (ESI): $m / z$ [M+H] $]^{+}$calcd for $\mathrm{C}_{18} \mathrm{H}_{31} \mathrm{O}_{4}: 311.2217$; found: 311.2219 .

(Z,Z)-Dodeca-5,7-diene-5,8-diyl diheptanoate (4q)

Colorless oil; yield: $0.173 \mathrm{~g}$ (0.409 mmol; 82\%).

IR (film): 2956, 2930, 2860, 1752, 1643, 1467, 1433, 1379, 1210, 1127, $1049,937,829,727 \mathrm{~cm}^{-1}$.

${ }^{1} \mathrm{H}$ NMR ( $\left.\mathrm{CDCl}_{3}, 300 \mathrm{MHz}\right): \delta 5.64(\mathrm{~s}, 2 \mathrm{H},=\mathrm{CH}), 2.46\left(\mathrm{t}, J=7.5 \mathrm{~Hz}, 4 \mathrm{H}, \mathrm{CH}_{2}\right)$, $2.27\left(\mathrm{t}, J=6.9 \mathrm{~Hz}, 4 \mathrm{H}, \mathrm{CH}_{2}\right), 1.77-1.67\left(\mathrm{~m}, 4 \mathrm{H}, \mathrm{CH}_{2}\right), 1.46-1.34(\mathrm{~m}, 20 \mathrm{H}$, $\left.\mathrm{CH}_{2}\right), 0.93-0.89\left(\mathrm{~m}, 12 \mathrm{H}, \mathrm{CH}_{3}\right)$.

${ }^{13} \mathrm{C} \mathrm{NMR}\left(\mathrm{CDCl}_{3}, 75 \mathrm{MHz}\right): \delta=171.5,149.8,109.0,34.3,33.4,31.4,28.8$, 28.7, 25.1, 22.5, 22.1, 14.0, 13.8 .

HRMS (ESI): $m / z[\mathrm{M}+\mathrm{Na}]^{+}$calcd for $\mathrm{C}_{26} \mathrm{H}_{46} \mathrm{O}_{4} \mathrm{Na}$ : 445.3288; found: 445.3287 .

\section{(Z,Z)-Dodeca-5,7-diene-5,8-diyl dioctanoate (4r)}

White solid; yield: $0.185 \mathrm{~g}$ (0.410 mmol; $82 \%)$; $\mathrm{mp} \mathrm{47-49}{ }^{\circ} \mathrm{C}$.

IR (KBr): 2927, 2871, 1742, 1641, 1468, 1418, 1380, 1321, 1271, 1224, $1154,1123,938,877,842,770,720,580,481 \mathrm{~cm}^{-1}$.

${ }^{1} \mathrm{H}$ NMR $\left(\mathrm{CDCl}_{3}, 300 \mathrm{MHz}\right): \delta 5.64(\mathrm{~s}, 2 \mathrm{H},=\mathrm{CH}), 2.46\left(\mathrm{t}, J=7.2 \mathrm{~Hz}, 4 \mathrm{H}, \mathrm{CH}_{2}\right)$, $2.27\left(\mathrm{t}, J=6.9 \mathrm{~Hz}, 4 \mathrm{H}, \mathrm{CH}_{2}\right), 1.77-1.67\left(\mathrm{~m}, 4 \mathrm{H}, \mathrm{CH}_{2}\right), 1.46-1.32(\mathrm{~m}, 24 \mathrm{H}$, $\left.\mathrm{CH}_{2}\right), 0.91\left(\mathrm{t}, J=6.9 \mathrm{~Hz}, 12 \mathrm{H}, \mathrm{CH}_{3}\right)$.

${ }^{13} \mathrm{C} \mathrm{NMR}\left(\mathrm{CDCl}_{3}, 75 \mathrm{MHz}\right): \delta=171.5,149.8,109.0,34.3,33.4,31.7,29.1$, 28.9, 28.7, 25.1, 22.6, 22.1, 14.0, 13.8 .

HRMS (ESI): $m / z[\mathrm{M}+\mathrm{Na}]^{+}$calcd for $\mathrm{C}_{28} \mathrm{H}_{50} \mathrm{O}_{4} \mathrm{Na}$ : 473.3601; found: 473.3595 .

\section{(Z,Z)-Dodeca-5,7-diene-5,8-diyl bis(3-bromopropanoate) (4s)}

White solid; yield: $0.175 \mathrm{~g}$ (0.374 mmol; 75\%); mp 66-68 ${ }^{\circ} \mathrm{C}$.

IR (KBr): 2960, 2941, 2874, 1747, 1643, 1467, 1427, 1336, 1285, 1217, $1132,1109,952,884,816,668,580,548 \mathrm{~cm}^{-1}$.

${ }^{1} \mathrm{H} \mathrm{NMR}\left(\mathrm{CDCl}_{3}, 400 \mathrm{MHz}\right): \delta 5.74(\mathrm{~s}, 2 \mathrm{H},=\mathrm{CH}), 3.65(\mathrm{t}, J=6.4 \mathrm{~Hz}, 4 \mathrm{H}$, $\mathrm{CH}_{2} \mathrm{Br}$ ), 3.08 (t, J = 6.4 Hz, $4 \mathrm{H}, \mathrm{CH}_{2} \mathrm{CH}_{2} \mathrm{Br}$ ), 2.27 (t, J = $7.6 \mathrm{~Hz}, 4 \mathrm{H}, \mathrm{CH}_{2}$ ), 1.46-1.40 (m, $\left.4 \mathrm{H}_{1} \mathrm{CH}_{2}\right), 1.35-1.29\left(\mathrm{~m}, 4 \mathrm{H}_{1} \mathrm{CH}_{2}\right), 0.89(\mathrm{t}, J=7.2 \mathrm{~Hz}, 6 \mathrm{H}$, $\left.\mathrm{CH}_{3}\right)$.

${ }^{13} \mathrm{C} \mathrm{NMR}\left(\mathrm{CDCl}_{3}, 101 \mathrm{MHz}\right): \delta=168.1,149.8,109.3,37.7,33.3,28.7,25.8$, $22.1,13.8$.

HRMS (ESI): $m / z$ [M+Na] $]^{+}$calcd for $\mathrm{C}_{18} \mathrm{H}_{28} \mathrm{O}_{4} \mathrm{Br} 2 \mathrm{Na}$ : 489.0247; found: 489.0247 .

(Z,Z)-Dodeca-5,7-diene-5,8-diyl bis(3-cyclopentylpropanoate) (4t) 


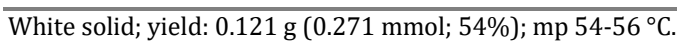

IR (KBr): 2951, 2868, 1740, 1649, 1467, 1420, 1381, 1355, 1303, 1262 , $1153,1130,999,930,883,829,731,563,441 \mathrm{~cm}^{-1}$.

${ }^{1} \mathrm{H} \mathrm{NMR}\left(\mathrm{CDCl}_{3}, 300 \mathrm{MHz}\right): \delta 5.63(\mathrm{~s}, 2 \mathrm{H},=\mathrm{CH}), 2.47\left(\mathrm{t}, J=7.8 \mathrm{~Hz}, 4 \mathrm{H}, \mathrm{CH}_{2}\right)$, $2.26\left(\mathrm{t}, J=6.9 \mathrm{~Hz}, 4 \mathrm{H}, \mathrm{CH}_{2}\right), 1.82-1.15\left(\mathrm{~m}, 30 \mathrm{H}, \mathrm{CH}_{2}+\mathrm{CH}\right), 0.90(\mathrm{t}, J=7.2$ $\left.\mathrm{Hz}, 6 \mathrm{H}, \mathrm{CH}_{3}\right)$.

${ }^{13} \mathrm{C} \mathrm{NMR}\left(\mathrm{CDCl}_{3}, 75 \mathrm{MHz}\right): \delta=171.5,149.8,109.0,39.6,33.6,33.3,32.4$, $31.3,28.7,25.1,22.1,13.8$.

HRMS (ESI): $m / z[\mathrm{M}+\mathrm{Na}]^{+}$calcd for $\mathrm{C}_{28} \mathrm{H}_{46} \mathrm{O}_{4} \mathrm{Na}$ : 469.3288; found: 469.3287.

\section{(Z,Z)-Dodeca-5,7-diene-5,8-diyl bis(2-phenylacetate) (4u)}

White solid; yield: $0.167 \mathrm{~g}$ (0.384 mmol; 77\%); mp 56-58 ${ }^{\circ} \mathrm{C}$.

IR (KBr): 2951, 2848, 1733, 1652, 1454, 1327, 1298, 1231, 1118, 1052 , $944,876,809,717,692,550,479 \mathrm{~cm}^{-1}$.

${ }^{1} \mathrm{H} \mathrm{NMR}\left(\mathrm{CDCl}_{3}, 300 \mathrm{MHz}\right): \delta$ 7.37-7.30 (m, $\left.10 \mathrm{H}, \mathrm{Ar}-\mathrm{H}\right), 5.30(\mathrm{~s}, 2 \mathrm{H},=\mathrm{CH})$, $3.72\left(\mathrm{~s}, 4 \mathrm{H}, \mathrm{CH}_{2}\right), 2.11\left(\mathrm{t}, J=7.2 \mathrm{~Hz}, 4 \mathrm{H}, \mathrm{CH}_{2}\right), 1.25-1.21\left(\mathrm{~m}, 8 \mathrm{H}, \mathrm{CH}_{2}\right), 0.86$ $\left(\mathrm{t}, J=6.6 \mathrm{~Hz}, 6 \mathrm{H}, \mathrm{CH}_{3}\right)$.

${ }^{13} \mathrm{C} \mathrm{NMR}\left(\mathrm{CDCl}_{3}, 75 \mathrm{MHz}\right): \delta=169.1,149.8,133.7,129.3,128.7,127.3$, 108.9, 41.6, 33.1, 28.5, 22.1, 13.8 .

HRMS (ESI): $m / z$ [M+Na] ${ }^{+}$calcd for $\mathrm{C}_{28} \mathrm{H}_{34} \mathrm{O}_{4} \mathrm{Na}$ : 457.2349; found: 457.2347.

\section{(Z,Z)-Dodeca-5,7-diene-5,8-diyl bis(2-methylpropanoate) (4v)}

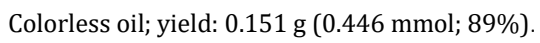

IR (film): 2959, 2934, 2874, 1753, 1644, 1469, 1386, 1343, 1233, 1182, $1120,1095,1052,935,828,748 \mathrm{~cm}^{-1}$.

${ }^{1} \mathrm{H} \mathrm{NMR}\left(\mathrm{CDCl}_{3}, 300 \mathrm{MHz}\right): \delta 5.64(\mathrm{~s}, 2 \mathrm{H},=\mathrm{CH}), 2.70(\mathrm{sept}, J=6.9 \mathrm{~Hz}, 2 \mathrm{H}$ $\mathrm{CH}), 2.27\left(\mathrm{t}, J=6.9 \mathrm{~Hz}, 4 \mathrm{H}, \mathrm{CH}_{2}\right), 1.43-1.32\left(\mathrm{~m}, 8 \mathrm{H}, \mathrm{CH}_{2}\right), 1.28(\mathrm{~d}, J=6.9$ $\left.\mathrm{Hz}, 12 \mathrm{H}, \mathrm{CH}_{3}\right), 0.90\left(\mathrm{t}, J=7.2 \mathrm{~Hz}, 6 \mathrm{H}^{\mathrm{C}} \mathrm{CH}_{3}\right)$.

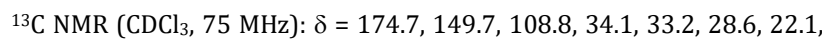
19.0, 13.8.

HRMS (ESI): $m / z[\mathrm{M}+\mathrm{Na}]^{+}$calcd for $\mathrm{C}_{20} \mathrm{H}_{34} \mathrm{O}_{4} \mathrm{Na}$ : 361.2349; found: 361.2345 .

\section{(Z,Z)-Dodeca-5,7-diene-5,8-diyl bis(2,2-dimethylpropanoate) (4w)}

Colorless oil; yield: $0.079 \mathrm{~g}$ (0.215 mmol; 43\%).

IR (film): 2959, 2933, 2873, 1747, 1645, 1480, 1462, 1396, 1369, 1263 , $1114,1029,938,843,761 \mathrm{~cm}^{-1}$.

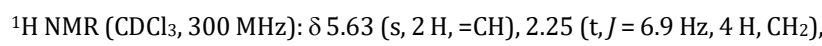
1.45-1.31 (m, $\left.8 \mathrm{H}, \mathrm{CH}_{2}\right), 1.27\left(\mathrm{~s}, 18 \mathrm{H}_{2} \mathrm{CH}_{3}\right), 0.90\left(\mathrm{t}, J=6.9 \mathrm{~Hz}, 6 \mathrm{H}, \mathrm{CH}_{3}\right)$.

${ }^{13} \mathrm{C} \mathrm{NMR}\left(\mathrm{CDCl}_{3}, 75 \mathrm{MHz}\right): \delta=176.1,149.7,108.8,39.1,33.1,28.5,27.2$, $22.0,13.8$.

HRMS (ESI): $m / z$ [M+Na] ${ }^{+}$calcd for $\mathrm{C}_{22} \mathrm{H}_{38} \mathrm{O}_{4} \mathrm{Na}$ : 389.2662; found: 389.2655 .

\section{(Z,Z)-Dodeca-5,7-diene-5,8-diyl dicyclopropanecarboxylate (4x)}

White solid; yield: $0.140 \mathrm{~g}$ (0.418 mmol; 84\%); mp 64-67 ${ }^{\circ} \mathrm{C}$.

IR (KBr): 2940, 2870, 1734, 1646, 1456, 1445, 1382, 1307, 1266, 1143, $1124,1098,1029,932,848,824,737,695,633,557,473 \mathrm{~cm}^{-1}$.

${ }^{1} \mathrm{H} \mathrm{NMR}\left(\mathrm{CDCl}_{3}, 300 \mathrm{MHz}\right): \delta 5.65(\mathrm{~s}, 2 \mathrm{H},=\mathrm{CH}), 2.27\left(\mathrm{t}, J=6.9 \mathrm{~Hz}, 4 \mathrm{H}, \mathrm{CH}_{2}\right)$, 1.75-1.72 (m, $2 \mathrm{H}, \mathrm{CH}), 1.46-1.30\left(\mathrm{~m}, 8 \mathrm{H}, \mathrm{CH}_{2}\right), 1.13-1.10\left(\mathrm{~m}, 4 \mathrm{H}, \mathrm{CH}_{2}\right)$, 0.99-0.95 (m, $\left.4 \mathrm{H}, \mathrm{CH}_{2}\right), 0.91\left(\mathrm{t}, J=7.2 \mathrm{~Hz}, 6 \mathrm{H}, \mathrm{CH}_{3}\right)$.

${ }^{13} \mathrm{C} \mathrm{NMR}\left(\mathrm{CDCl}_{3}, 75 \mathrm{MHz}\right): \delta=172.5,149.7,109.0,33.3,28.7,22.1,13.8$, 12.8, 8.8.

HRMS (ESI): $m / z[\mathrm{M}+\mathrm{Na}]^{+}$calcd for $\mathrm{C}_{20} \mathrm{H}_{30} \mathrm{O}_{4} \mathrm{Na}$ : 357.2036; found: 357.2034 .

(Z,Z)-Dodeca-5,7-diene-5,8-diyl dicyclohexanecarboxylate (4y)
White solid; yield: $0.176 \mathrm{~g}(0.420 \mathrm{mmol} ; 84 \%) ; \mathrm{mp} \mathrm{79-81}{ }^{\circ} \mathrm{C}$.

IR (KBr): 2929, 2858, 2669, 1749, 1643, 1451, 1376, 1311, 1241, 1211, $1152,1117,1018,937,896,870,831,743,694,612,547,488 \mathrm{~cm}^{-1}$.

${ }^{1} \mathrm{H}$ NMR $\left(\mathrm{CDCl}_{3}, 300 \mathrm{MHz}\right): \delta 5.63(\mathrm{~s}, 2 \mathrm{H},=\mathrm{CH}), 2.50-2.43(\mathrm{~m}, 2 \mathrm{H}, \mathrm{CH}), 2.25$ $\left(\mathrm{t}, J=6.3 \mathrm{~Hz}, 4 \mathrm{H}, \mathrm{CH}_{2}\right), 2.03-1.99\left(\mathrm{~m}, 4 \mathrm{H}, \mathrm{CH}_{2}\right), 1.79-1.32\left(\mathrm{~m}, 24 \mathrm{H}, \mathrm{CH}_{2}\right)$, $0.90\left(\mathrm{t}, J=6.9 \mathrm{~Hz}, 6 \mathrm{H}, \mathrm{CH}_{3}\right)$.

${ }^{13} \mathrm{C} \mathrm{NMR}\left(\mathrm{CDCl}_{3}, 75 \mathrm{MHz}\right): \delta=173.7,149.7,108.9,43.2,33.3,29.1,28.6$, 25.7, 25.4, 22.1, 13.9 .

HRMS (ESI): $m / z[\mathrm{M}+\mathrm{Na}]^{+}$calcd for $\mathrm{C}_{26} \mathrm{H}_{42} \mathrm{O}_{4} \mathrm{Na}$ : 441.2975; found: 441.2976

\section{(Z,Z)-Dodeca-5,7-diene-5,8-diyl dibenzoate (4z)}

White solid; yield: $0.112 \mathrm{~g}$ (0.275 mmol; 55\%); mp 159-161 ${ }^{\circ} \mathrm{C}$.

IR (KBr): 2955, 2926, 2861, 1724, 1652, 1600, 1452, 1284, 1257, 1238, $1174,1108,1064,1025,865,800,702,682,622 \mathrm{~cm}^{-1}$.

${ }^{1} \mathrm{H} \mathrm{NMR}\left(\mathrm{CDCl}_{3}, 300 \mathrm{MHz}\right): \delta 8.18(\mathrm{~d}, J=7.5 \mathrm{~Hz}, 4 \mathrm{H}, \mathrm{Ar}-\mathrm{H}), 7.68-7.51(\mathrm{~m}, 6$ $\mathrm{H}, \mathrm{Ar}-\mathrm{H}), 5.86(\mathrm{~s}, 2 \mathrm{H},=\mathrm{CH}), 2.38\left(\mathrm{t}, J=7.3 \mathrm{~Hz}, 4 \mathrm{H}, \mathrm{CH}_{2}\right), 1.49-1.44(\mathrm{~m}, 4 \mathrm{H}$, $\left.\mathrm{CH}_{2}\right), 1.36-1.29\left(\mathrm{~m}, 4 \mathrm{H}, \mathrm{CH}_{2}\right), 0.86\left(\mathrm{t}, J=7.0 \mathrm{~Hz}, 6 \mathrm{H}, \mathrm{CH}_{3}\right)$.

${ }^{13} \mathrm{C} \mathrm{NMR}\left(\mathrm{CDCl}_{3}, 75 \mathrm{MHz}\right): \delta=164.3,150.2,133.5,130.1,129.5,128.6$, 109.6, 33.5, 28.8, 22.2, 13.8 .

HRMS (ESI): $m / z$ [M+Na] ${ }^{+}$calcd for $\mathrm{C}_{26} \mathrm{H}_{30} \mathrm{O}_{4} \mathrm{Na}$ : 429.2036; found: 429.2039.

\section{(Z,Z)-Dodeca-5,7-diene-5,8-diyl bis(4-methoxybenzoate) (4aa)}

White solid; yield: $0.124 \mathrm{~g}$ (0.266 mmol; 53\%); $\mathrm{mp} 170-172{ }^{\circ} \mathrm{C}$.

IR (KBr): 2956, 2931, 2872, 1717, 1644, 1605, 1511, 1461, 1321, 1288, $1253,1165,1103,1074,1028,844,766,694,599 \mathrm{~cm}^{-1}$.

${ }^{1} \mathrm{H} \mathrm{NMR}\left(\mathrm{CDCl}_{3}, 300 \mathrm{MHz}\right): \delta 8.12(\mathrm{~d}, J=8.9 \mathrm{~Hz}, 4 \mathrm{H}, \mathrm{Ar}-\mathrm{H}), 7.01(\mathrm{~d}, J=8.9$ $\mathrm{Hz}, 4 \mathrm{H}, \mathrm{Ar}-\mathrm{H}), 5.83(\mathrm{~s}, 2 \mathrm{H},=\mathrm{CH}), 3.92\left(\mathrm{~s}, 6 \mathrm{H}, \mathrm{OCH}_{3}\right), 2.36(\mathrm{t}, J=7.2 \mathrm{~Hz}, 4$ $\left.\mathrm{H}, \mathrm{CH}_{2}\right), 1.50-1.40\left(\mathrm{~m}, 4 \mathrm{H}, \mathrm{CH}_{2}\right), 1.38-1.26\left(\mathrm{~m}, 4 \mathrm{H}, \mathrm{CH}_{2}\right), 0.86(\mathrm{t}, J=6.9 \mathrm{~Hz}$, $\left.6 \mathrm{H} \mathrm{CH}_{3}\right)$.

${ }^{13} \mathrm{C} \mathrm{NMR}\left(\mathrm{CDCl}_{3}, 75 \mathrm{MHz}\right): \delta=164.1,163.8,150.1,132.2,121.9,113.8$, 109.6, 55.5, 33.5, 28.8, 22.2, 13.8 .

HRMS (ESI): $m / z[\mathrm{M}+\mathrm{Na}]^{+}$calcd for $\mathrm{C}_{28} \mathrm{H}_{34} \mathrm{O}_{6} \mathrm{Na}$ : 489.2253; found: 489.2248 .

\section{(Z,Z)-Dodeca-5,7-diene-5,8-diyl bis(4-fluorobenzoate) (4ab)}

White solid; yield: $0.135 \mathrm{~g}$ (0.305 mmol; 61\%); mp 165-167 ${ }^{\circ} \mathrm{C}$.

IR (KBr): 3079, 2954, 2930, 2872, 1728, 1646, 1603, 1507, 1414, 1280, $1234,1156,1104,1072,1011,858,839,764,686,597 \mathrm{~cm}^{-1}$.

${ }^{1} \mathrm{H} \mathrm{NMR}\left(\mathrm{CDCl}_{3}, 300 \mathrm{MHz}\right): \delta 8.18(\mathrm{dd}, J=8.4$ and $5.4 \mathrm{~Hz}, 4 \mathrm{H}, \mathrm{Ar}-\mathrm{H}), 7.20$ $(\mathrm{t}, J=8.4 \mathrm{~Hz}, 4 \mathrm{H}, \mathrm{Ar}-\mathrm{H}), 5.81(\mathrm{~s}, 2 \mathrm{H},=\mathrm{CH}), 2.36\left(\mathrm{t}, J=7.2 \mathrm{~Hz}, 4 \mathrm{H}, \mathrm{CH}_{2}\right)$, 1.47-1.40 (m, $\left.4 \mathrm{H}, \mathrm{CH}_{2}\right), 1.38-1.28\left(\mathrm{~m}, 4 \mathrm{H}, \mathrm{CH}_{2}\right), 0.86(\mathrm{t}, J=7.2 \mathrm{~Hz}, 6 \mathrm{H}$, $\left.\mathrm{CH}_{3}\right)$.

${ }^{13} \mathrm{C} \mathrm{NMR}\left(\mathrm{CDCl}_{3}, 75 \mathrm{MHz}\right): \delta=166.1(\mathrm{~d}, J=254.9 \mathrm{~Hz}), 163.3,150.1,132.6$ (d, $J=9.4 \mathrm{~Hz}$ ), 125.6, 115.8 (d, $J=22.0 \mathrm{~Hz}$ ), 109.5, 33.4, 28.8, 22.1, 13.8 .

${ }^{19} \mathrm{~F} \mathrm{NMR}\left(\mathrm{CDCl}_{3}, 282 \mathrm{MHz}\right): \delta-104.6$.

HRMS (ESI): $m / z[\mathrm{M}+\mathrm{Na}]^{+}$calcd for $\mathrm{C}_{26} \mathrm{H}_{28} \mathrm{O}_{4} \mathrm{~F}_{2} \mathrm{Na}$ : 465.1848; found: 465.1845 .

\section{(Z,Z)-Dodeca-5,7-diene-5,8-diyl bis(4-vinylbenzoate) (4ac)}

White solid; yield: $0.147 \mathrm{~g} \mathrm{(0.320} \mathrm{mmol;} \mathrm{64 \% );} \mathrm{mp} \mathrm{140-142}{ }^{\circ} \mathrm{C}$.

IR (KBr): 2953, 2929, 2871, 1721, 1646, 1606, 1466, 1406, 1256, 1236, $1177,1104,1076,1016,932,855,780,709,599 \mathrm{~cm}^{-1}$.

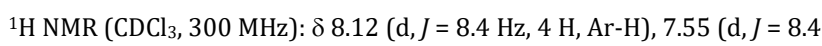
$\mathrm{Hz}, 4 \mathrm{H}, \mathrm{Ar}-\mathrm{H}$ ), 6.81 (dd, $J=17.4$ and $11.1 \mathrm{~Hz}, 2 \mathrm{H},=\mathrm{CH}$ ), 5.93 (d, $J=17.4$ $\left.\mathrm{Hz}, 2 \mathrm{H},=\mathrm{CH}_{2}\right), 5.84(\mathrm{~s}, 2 \mathrm{H},=\mathrm{CH}), 5.45\left(\mathrm{~d}, J=11.1 \mathrm{~Hz}, 2 \mathrm{H},=\mathrm{CH}_{2}\right), 2.37(\mathrm{t}, J$ $\left.=7.2 \mathrm{~Hz}, 4 \mathrm{H}, \mathrm{CH}_{2}\right), 1.50-1.43\left(\mathrm{~m}, 4 \mathrm{H}, \mathrm{CH}_{2}\right), 1.40-1.26\left(\mathrm{~m}, 4 \mathrm{H}, \mathrm{CH}_{2}\right), 0.86$ $\left(\mathrm{t}, J=7.2 \mathrm{~Hz}, 6 \mathrm{H}, \mathrm{CH}_{3}\right)$. 
${ }^{13} \mathrm{C}$ NMR $\left(\mathrm{CDCl}_{3}, 75 \mathrm{MHz}\right): \delta=163.9,150.2,142.5,135.9,130.4,128.6$, $126.3,116.9,109.5,33.5,28.8,22.2,13.8$.

HRMS (ESI): $m / z$ [M+H] $]^{+}$calcd for $\mathrm{C}_{30} \mathrm{H}_{35} \mathrm{O}_{4}: 459.2530$; found: 459.2528 .

\section{(Z,Z)-Dodeca-5,7-diene-5,8-diyl bis(3-methylbenzoate) (4ad)}

White solid; yield: $0.126 \mathrm{~g} \mathrm{(0.290} \mathrm{mmol;} \mathrm{58 \% );} \mathrm{mp} 162-164{ }^{\circ} \mathrm{C}$.

IR (KBr): 2956, 2928, 2871, 1726, 1611, 1466, 1422, 1286, 1262, 1184, $1107,916,882,810,778,737,679,622 \mathrm{~cm}^{-1}$.

${ }^{1} \mathrm{H} \mathrm{NMR}\left(\mathrm{CDCl}_{3}, 300 \mathrm{MHz}\right): \delta 8.01-7.98(\mathrm{~m}, 4 \mathrm{H}, \mathrm{Ar}-\mathrm{H}), 7.49-7.40(\mathrm{~m}, 4 \mathrm{H}$, $\mathrm{Ar}-\mathrm{H}), 5.88(\mathrm{~s}, 2 \mathrm{H},=\mathrm{CH}), 2.48\left(\mathrm{~s}, 6 \mathrm{H}, \mathrm{CH}_{3}\right), 2.40\left(\mathrm{t}, J=7.2 \mathrm{~Hz}, 4 \mathrm{H}, \mathrm{CH}_{2}\right)$, 1.51-1.46 (m, $\left.4 \mathrm{H}, \mathrm{CH}_{2}\right), 1.38-1.31\left(\mathrm{~m}, 4 \mathrm{H}, \mathrm{CH}_{2}\right), 0.88(\mathrm{t}, J=7.2 \mathrm{~Hz}, 6 \mathrm{H}$ $\left.\mathrm{CH}_{3}\right)$.

${ }^{13} \mathrm{C}$ NMR $\left(\mathrm{CDCl}_{3}, 75 \mathrm{MHz}\right): \delta=164.5,150.2,138.5,134.3,130.6,129.5$, $128.5,127.2,109.6,33.5,28.8,22.2,21.3,13.8$

HRMS (ESI): $m / z$ [M+Na $]^{+}$calcd for $\mathrm{C}_{28} \mathrm{H}_{34} \mathrm{O}_{4} \mathrm{Na}: 457.2349$; found: 457.2348.

\section{(Z,Z)-Dodeca-5,7-diene-5,8-diyl bis(thiophene-2-carboxylate) (4ae)}

White solid; yield: $0.090 \mathrm{~g}(0.215 \mathrm{mmol} ; 43 \%) ; \mathrm{mp} 92-94^{\circ} \mathrm{C}$.

IR (KBr): 2954, 2928, 2861, 1725, 1643, 1519, 1467, 1413, 1359, 1242, $1217,1101,1063,861,820,777,743,711,603,541 \mathrm{~cm}^{-1}$.

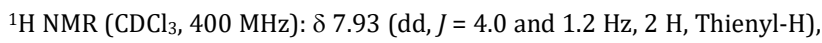
$7.66(\mathrm{dd}, J=5.2$ and $1.2 \mathrm{~Hz}, 2 \mathrm{H}$, Thienyl-H), 7.18 (dd, $J=5.2$ and $4.0 \mathrm{~Hz}, 2$ $\mathrm{H}$, Thienyl-H), $5.85(\mathrm{~s}, 2 \mathrm{H},=\mathrm{CH}), 2.38\left(\mathrm{t}, J=7.2 \mathrm{~Hz}, 4 \mathrm{H}, \mathrm{CH}_{2}\right), 1.50-1.45(\mathrm{~m}$, $\left.4 \mathrm{H}, \mathrm{CH}_{2}\right), 1.43-1.28\left(\mathrm{~m}, 4 \mathrm{H}, \mathrm{CH}_{2}\right), 0.87$ (t, J $\left.=7.2 \mathrm{~Hz}, 6 \mathrm{H}, \mathrm{CH}_{3}\right)$.

${ }^{13} \mathrm{C}$ NMR $\left(\mathrm{CDCl}_{3}, 101 \mathrm{MHz}\right): \delta=159.8,149.9,134.4,133.3,132.9,128.0$, 109.7, 33.4, 28.8, 22.2, 13.8.

HRMS (ESI): $m / z$ [M+Na] ${ }^{+}$calcd for $\mathrm{C}_{22} \mathrm{H}_{26} \mathrm{O}_{4} \mathrm{~S}_{2} \mathrm{Na}$ : 441.1165; found: 441.1160 .

(Z,Z)-Dodeca-5,7-diene-5,8-diyl bis(tetrahydrofuran-2-carboxylate) (4af)

Colorless oil; yield: $0.079 \mathrm{~g}$ ( $0.200 \mathrm{mmol}$; $40 \%)$.

IR (film): 2956, 2932, 2873, 1767, 1645, 1466, 1348, 1245, 1154, 1129, $1084,964,930,883,839,744 \mathrm{~cm}^{-1}$.

${ }^{1} \mathrm{H} \mathrm{NMR}\left(\mathrm{CDCl}_{3}, 300 \mathrm{MHz}\right): \delta 5.64(\mathrm{~s}, 2 \mathrm{H},=\mathrm{CH}), 4.60(\mathrm{dd}, J=8.1$ and $5.1 \mathrm{~Hz}$, $2 \mathrm{H}, \mathrm{OCH}), 4.07-3.95\left(\mathrm{~m}, 4 \mathrm{H}, \mathrm{OCH}_{2}\right), 2.41-2.29\left(\mathrm{~m}, 2 \mathrm{H}, \mathrm{OCHCH}_{2}\right), 2.27(\mathrm{t}, J$ $\left.=7.2 \mathrm{~Hz}, 4 \mathrm{H}, \mathrm{CH}_{2}\right), 2.12-2.09\left(\mathrm{~m}, 2 \mathrm{H}, \mathrm{OCH}_{2} \mathrm{CH}_{2}\right), 2.02-1.96\left(\mathrm{~m}, 4 \mathrm{H}, \mathrm{CH}_{2}\right)$, $1.42-1.30\left(\mathrm{~m}, 8 \mathrm{H}, \mathrm{CH}_{2}\right), 0.88\left(\mathrm{t}, J=7.2 \mathrm{~Hz}, 6 \mathrm{H}^{-\mathrm{CH}_{3}}\right)$.

${ }^{13} \mathrm{C}$ NMR $\left(\mathrm{CDCl}_{3}, 75 \mathrm{MHz}\right): \delta=171.0,149.6,109.0,76.5,69.5,33.1,30.4$, 28.6, 25.3, 22.1, 13.8 .

HRMS (ESI): $m / z$ [M+H] $]^{+}$calcd for $\mathrm{C}_{22} \mathrm{H}_{35} \mathrm{O}_{6}: 395.2428$; found: 395.2430 .

\section{(Z,Z)-Dodeca-5,7-diene-5,8-diyl (E,E)-bis(3-phenylacrylate) (4ag)}

White solid; yield: $0.101 \mathrm{~g}(0.220 \mathrm{mmol} ; 44 \%)$; mp $115-117{ }^{\circ} \mathrm{C}$.

IR (KBr): 2960, 2931, 2859, 1722, 1634, 1579, 1467, 1423, 1326, 1308, $1271,1141,1112,979,860,766,706,680,566,481 \mathrm{~cm}^{-1}$.

${ }^{1} \mathrm{H} \mathrm{NMR}\left(\mathrm{CDCl}_{3}, 300 \mathrm{MHz}\right): \delta 7.83(\mathrm{~d}, J=15.9 \mathrm{~Hz}, 2 \mathrm{H},=\mathrm{CH}), 7.61(\mathrm{br}, 4 \mathrm{H}$, Ar-H), $7.45(\mathrm{br}, 6 \mathrm{H}, \mathrm{Ar}-\mathrm{H}), 6.58(\mathrm{~d}, J=15.9 \mathrm{~Hz}, 2 \mathrm{H},=\mathrm{CH}), 5.80(\mathrm{~s}, 2 \mathrm{H},=\mathrm{CH})$, $2.36\left(\mathrm{t}, J=6.9 \mathrm{~Hz}, 4 \mathrm{H}, \mathrm{CH}_{2}\right), 1.53-1.29\left(\mathrm{~m}, 8 \mathrm{H}, \mathrm{CH}_{2}\right), 0.90(\mathrm{t}, J=7.2 \mathrm{~Hz}, 6 \mathrm{H}$, $\mathrm{CH}_{3}$ ).

${ }^{13} \mathrm{C}$ NMR $\left(\mathrm{CDCl}_{3}, 75 \mathrm{MHz}\right): \delta=164.6,150.0,146.3,134.2,130.7,129.0$, $128.3,117.2,109.4,33.5,28.8,22.2,13.9$.

HRMS (ESI): $m / z$ [M+Na] ${ }^{+}$calcd for $\mathrm{C}_{30} \mathrm{H}_{34} \mathrm{O}_{4} \mathrm{Na}$ : 481.2349; found: 481.2347.

\section{Funding Information}

This work was supported by the Spanish MINECO (CTQ2016-75986-P) and the University of Oviedo (PAPI-18-GR-2011-0032).

\section{Acknowledgment}

We thank Dr. Pedro J. González-Liste and Dr. Sergio E. García-Garrido for helpful discussions and comments.

\section{Supporting Information}

YES (this text will be updated with links prior to publication)

\section{Primary Data}

NO (this text will be deleted prior to publication)

\section{References}

(1) See, for example: (a) Bruneau, C.; Neveux, M.; Kabouche, Z.; Ruppin, C.; Dixneuf, P. H. Synlett 1991, 755. (b) Takeno, M.; Kikuchi, S.; Morita, K.-I.; Nishiyama, Y.; Ishii, Y. J. Org. Chem. 1995, 60, 4974. (c) Dang, H.-S.; Roberts, B. P. Chem. Commun. 1996, 2201. (d) Bonnarme, V.; Bachmann, C.; Cousson, A.; Mondon, M.; Gesson, J.-P. Tetrahedron 1999, 55, 433. (e) Urabe, H.; Suzuki, D.; Sasaki, M.; Sato, F. J. Am. Chem. Soc. 2003, 125, 4036. (f) Gooßen, L. J.; Paetzold, J. Angew. Chem. Int. Ed. 2004, 43, 1095. (g) Isambert, N.; Cruz, M.; Arévalo, M. J.; Gómez, E.; Lavilla, R. Org. Lett. 2007, 9, 4199. (h) Magens, S.; Ertelt, M.; Jatsch, A.; Plietker, B. Org. Lett. 2008, 10, 53. (i) Onishi, Y.; Yoneda, Y.; Nishimoto, Y.; Yasuda, M.; Baba, A. Org. Lett. 2012, 14, 5788. (j) Abrams, M. L.; Foarta, F.; Landis, C. R. J. Am. Chem. Soc. 2014, 136, 14583. (k) Yin, Z.; Liu, Z.; Huang, Z.; Chu, Y.; Chu, Z.; Hu, J.; Gao, L.; Song, Z. Org. Lett. 2015, 17, 1553. (l) Foarta, F.; Landis, C. R. J. Org. Chem. 2016, 81, 11250. (m) Jena, R. K.; Das, U. K.; Ghorai, A.; Bhattacharjee, M. Eur. J. Org. Chem. 2016, 6015. (n) Masutomi, K.; Sugiyama, H.; Uekasa, H.; Shibata, Y.; Tanaka, K. Angew. Chem. Int. Ed. 2016, 55, 15373. (o) Schmitz, C.; Holthusen, K.; Leitner, W.; Franciò, G. ACS Catal. 2016, 6, 1584. (p) Krause, T.; Baader, S.; Erb, B.; Gooßen, L. J. Nat. Commun. 2016, 7, 11732. (q) Geibel, I.; Dierks, A.; Müller, T.; Christoffers, J. Chem. Eur. J. 2017, 23, 7245. (r) Calmanti, R.; Galvan M.; Amadio, E.; Perosa, A.; Selva, M. ACS Sustainable Chem. Eng. 2018, 6, 3964.

(2) See, for example: (a) Burk, M. J.; Kalberg, C. S.; Pizzano, A. J. Am Chem. Soc. 1998, 120, 4345. (b)Tang, W.; Liu, D.; Zhang, X. Org. Lett. 2003, 5, 205. (c) Reetz, M. T.; Gooßen, L. J.; Meiswinkel, A.; Paetzold, J.; Jensen, J. F. Org. Lett. 2003, 5, 3099. (d) Zhang, X.; Huang, K.; Hou, G.; Cao, B.; Zhang, X. Angew. Chem. Int. Ed. 2010, 49, 6421. (e) Mamone, P.; Grünberg, M. F.; Fromm, A.; Khan, B. A. Gooßen, L. J. Org. Lett. 2012, 14, 3716. (f) Liu, Y.; Wang, Z.; Ding, K. Tetrahedron 2012, 68, 7581. (g) Núñez-Rico, J. L.; Etayo, P.; Fernández-Pérez, H.; Vidal-Ferran, A. Adv. Synth. Catal. 2012, 354, 3025. (h) Konrad, T. M.; Schmitz, P.; Leitner, W.; Franciò, G. Chem. Eur. J. 2013, 19, 13299. (i) Kleman, P.; González-Liste, P. J.; GarcíaGarrido, S. E.; Cadierno, V.; Pizzano, A. Chem. Eur. J. 2013, 19, 16209. (j) Arena, G.; Barreca, G.; Carcone, L.; Cini, E.; Marras, G.; Nedden, H. G.; Rasparini, M.; Roseblade, S.; Russo, A.; Taddei, M.; Zanotti-Gerosa, A. Adv. Synth. Catal. 2013, 355, 1449. (k) Kleman, P.; González-Liste, P. J.; García-Garrido, S. E.; Cadierno, V.; Pizzano, A. ACS Catal. 2014, 4, 4398. (I) Jia, J.; Fan, D.; Zhang, J.; Zhang, Z.; Zhang, W. Adv. Synth. Catal. 2018, 360, 3793. (m) Liu, C.; Yuan, J.; Zhang, J.; Wang, Z.; Zhang, Z.; Zhang, W. Org. Lett. 2018, 20, 108.

(3) Zhang, C.; Chen, L.; Chen, K.; Wang, C.; Xu, Z.; Jiang, H.; Zhu, S. Org. Chem. Front. 2018, 5, 2510 and references cited therein.

(4) For reviews covering this topic, see: (a) Alonso, F.; Beletskaya, I. P.; Yus, M. Chem. Rev. 2004, 104, 3079. (b) Beller, M.; Seayard, J.; Tillack, A.; Jiao, H. Angew. Chem. Int. Ed. 2004, 43, 3368. (c) Gooßen, L. J.; Rodríguez, N.; Gooßen, K. Angew. Chem. Int. Ed. 2008, 47, 3100. (d) Hintermann, L. Top. Organomet. Chem. 2010, 31, 123. (e) Bruneau, C. Top. Organomet. Chem. 2011, 43, 203. (f) GonzálezListe, P. J.; Francos, J.; García-Garrido, S. E.; Cadierno, V. Arkivoc 2018, (ii), 17.

(5) For very recent examples, see: (a) Stark, M. J.; Tang, D. T.; Rath, N. P.; Bauer, E. B. Tetrahedron Lett. 2018, 59, 873. (b) Chen, J.-F.; Li, C. 
Org. Lett. 2018, 20, 6719. (c) Kukui, N.; Hinoue, T.; Yoshinosuke, U.; Satoh, T. Chem. Lett. 2018, 47, 141.

(6) (a) Cadierno, V.; Francos, J.; Gimeno, J. Organometallics 2011, 30, 852. (b) Tomás-Mendivil, E.; Toullec, P. Y.; Díez, J.; Conejero, S.; Michelet, V.; Cadierno, V. Org. Lett. 2012, 14, 2520. (c) TomásMendivil, E.; Toullec, P. Y.; Borge, J.; Conejero, S.; Michelet, V.; Cadierno, V. ACS Catal. 2013, 3, 3086. (d) González-Liste, P. J.; León, F.; Arribas, I.; Rubio, M.; García-Garrido, S. E.; Cadierno, V.; Pizzano, A. ACS Catal. 2016, 6, 3056. (e) González-Liste, P. J.; García-Garrido, S. E.; Cadierno, V. Org. Biomol. Chem. 2017, 15, 1670. (f) GonzálezListe, P. J.; Francos, J.; García-Garrido, S. E.; Cadierno, V.J. Org. Chem. 2017, 82, 1507. (g) León, F.; González-Liste, P. J.; García-Garrido, S. E.; Arribas, I.; Rubio, M.; Cadierno, V.; Pizzano, A. J. Org. Chem. 2017, $82,5852$.

(7) (a) For a review article covering homocoupling reactions of alkenyl halides, see: Stefani, H. A.; Guarezemini, A. S.; Cella, R. Tetrahedron 2010, 66, 7871. (b) For a relevant example of this reaction, see: Bach, A.; Lentz, D.; Luger, P.; Masserschmidt, M.; Olesch, C.; Patzschke, M. Angew. Chem. Int. Ed. 2002, 41, 296.

(8) All of them were obtained using different synthetic routes, not involving olefin-homocoupling reactions: (a) Lutz, R. E.; Reveley, W. G.; Mattox, V. R. J. Am. Chem. Soc. 1941, 63, 3171. (b) Oku, A.; Urano, S.-I.; Nakaji, T.; Quing, G.; Abe, M. J. Org. Chem. 1992, 57, 2263. (c) van Saarloos, T. J.; Regeling, H.; Zwanenburg, B.; Chittenden, G. J. F. J. Carbohydr. Chem. 1995, 14, 1007. (d) Evidente, A.; Ricciardiello, G.; Andolfi, A.; Sabatini, M. A.; Ganassi, S.; Altomare, C.; Favilla, M.; Melck, D. J. Agric. Food Chem. 2008, 56, 3569. (e) Wilkerson-Hill, S. M.; Sawano, S.; Sarpong, R. J. Org. Chem. 2016, 81, 11132 .
(9) León, F.; Francos, J.; López-Serrano, J.; García-Garrido, S. E.; Cadierno, V.; Pizzano, A. Chem. Commun. 2019, 55, 786.

(10) Innitzer, A. Synlett 2005, 2405.

(11) Zhang, S.; Zhang, D.; Liebeskind, L. S. J. Org. Chem. 1997, 62, 2312.

(12) (a) Xu, J.; Burton, D. J. Tetrahedron Lett. 2002, 43, 4565. (b) Eddarir, S.; Rolando, C. J. Fluorine Chem. 2004, 125, 377. (c) Xu, J.; Burton, D. J. J. Fluorine Chem. 2007, 128, 71. (d) Barluenga, J.; Moriel, P.; Aznar, F.; Valdés, C. Org. Lett. 2007, 9, 275. (e) Batsanov, A. S.; Knowles, J. P.; Samsam, B.; Whiting, A. Adv. Synth. Catal. 2008, 350, 227.

(13) See, for example: (a) Semmelhack, M. F.; Helquist, P.; Jones, L. D.; Keller, L.; Mendelson, L.; Ryono, L. S.; Smith, J. G.; Stauffer, R. D. J. Am. Chem. Soc. 1981, 103, 6460. (b) Takagi, K.; Hayama, N.; Sasaki, K. Bull. Chem. Soc. Jpn. 1984, 57, 1887. (c) Sasaki, K.; Nakao, K.; Kobayashi, Y.; Sakai, M.; Uchino, N.; Sakakibara, Y.; Takagi, K. Bull. Chem. Soc. Jpn. 1993, 66, 2446. (d) Cannes, C.; Condon, S.; Durandetti, M.; Périchon, J.; Nédélec, J.-Y. J. Org. Chem. 2000, 65, 4575. (e) Rodriguez, J. C.; Tejedor, J. L.; Rumbero, A.; Canoira, L. Tetrahedron 2006, 62, 3075. (f) Rodríguez, J. G.; Díaz-Oliva, C. Tetrahedron 2009, 65, 2512. (g) Peng, J.; Liu, X.; Kishi, Y. Tetrahedron Lett. 2011, 52, 2172. (h) Liu, Y.; Xiao, S.; Qi, Y.; Du, F. Chem. Asian J. 2017, 12, 673.

(14) CCDC deposition number 1869873. The data can be obtained free of charge from The Cambridge Crystallographic Data Centre via www.ccdc.cam.ac.uk/getstructures.

(15) Kuchitsu, K.; Fukuyama, T.; Morino, Y.J. Mol. Struct. 1968, 1, 463.

(16) Takagi, K.; Mimura, H.; Inokawa, S. Bull. Chem. Soc. Jpn. 1984, 57, 3517.

(17) Barnett, K. W. J. Chem. Educ. 1974, 51, 422. 